\title{
Cost-sharing contract design in a low-carbon service supply chain
}

\author{
Peng $\mathrm{He}^{1}$, Yong He${ }^{1, *}$, Chunming (Victor) Shi ${ }^{2}$, Henry $\mathrm{Xu}^{3}$, Li Zhou ${ }^{4}$ \\ ${ }^{1}$ School of Economics and Management, Southeast University, Nanjing 210096 \\ ${ }^{2}$ Lazaridis School of Business and Economics, Wilfrid Laurier University, Waterloo, \\ N2L 3C5, Canada \\ ${ }^{3}$ UQ Business School, The University of Queensland, Brisbane, QLD 4072, Australia \\ ${ }^{4}$ Business School, University of Greenwich, London, UK
}

*Corresponding author: Yong He, Ph.D, Professor,

School of Economics and Management

Southeast University, Nanjing 210096, China

Tel: +8618905162198

E-mail address: hy@ @eu.edu.cn

Declaration of interest: Conflicts of interest: none

Acknowledgments: This work was supported by the National Natural

Science Foundation of China (Nos.71771053 and 71371003), the Key

Research and Development Plan (Modern Agriculture) of Jiangsu

Province (No. BE2018385), the Fundamental Research Funds for the

Central Universities and Postgraduate Research \& Practice Innovation

Program of Jiangsu Province (No. KYCX18_0197). 


\section{Highlights}

- Design the cost-sharing contract in a service supply chain

- Find the optimal service level, emission reduction and advertising efforts

- Analyze the impact of corporate social responsibility on contract design 


\title{
Cost-sharing contract design in a low-carbon service supply chain
}

\begin{abstract}
:
This paper investigates a service supply chain (SC) consisting of a service provider (SP) who is in charge of carbon emission reduction and service, and a service integrator (SI) who is responsible for low-carbon advertising, considering corporate social responsibility (CSR). Given that SP shares SI's advertising cost, SI may have three types of cost sharing decisions, namely, not sharing any cost of SP (contract PA), sharing SP's emission reduction cost (contract PAIE), or sharing SP's service cost (contract PAIS). We establish three differential game models to explore the optimal decisions, and identify the conditions under which SP and SI would provide positive participation rates. Our findings demonstrate that consumers' low-carbon preference, and chain members' marginal profits and CSR behaviors significantly influence the optimal solutions. Furthermore, we indicate that two-way contracts (contracts PAIE and PAIS) could benefit the entire service SC and its members. Specifically, SI prefers contract PAIE when SP's service cost efficiency is lower, whereas he would rather choose contract PAIS under a higher one. More importantly, contracts PAIS and PAIE would be the potential equilibrium contract when SI has a relatively high marginal profit. When it is sufficiently low, contracts PAIE and PA would be the possible equilibrium contract.
\end{abstract}

Keywords: Emission reduction, Cost-sharing contract, Corporate social responsibility, Service supply chain 


\section{Introduction}

Driven by environmental pollution threat to the survival and health of humankind, in recent decades, low-carbon service and manufacturing have increasingly become a hot research topic. Extant research has confirmed that carbon emission from manufacturing and service is a main source for global warming (Bazan, Jaber, \& Zanoni, 2016; Sayed, Hendry, \& Bell, 2017; Wang, Wei, \& Huang, 2016; Xia, Guo, Qin, Yue, \& Zhu, 2018; Yang, Ji, Wang, \& Wang, 2018). To curb carbon emission, many countries throughout the world have designed and implemented various low-carbon policies and regulations (e.g., Cap and Trade, Green Subsidy, Carbon Tax etc.). For example, Chinese government announced the goal to reduce its carbon emission per-unit GDP by $40-45 \%$ by the year 2020 compared to that in 2005 (Yang \& Chen, 2018). On the market side, with the improvement of people's environmental consciousness, an increasing number of environmentally friendly consumers are willing to pay extra for the low-carbon products and services (Achtnicht, 2012; Liu \& Song, 2017; Zhao, Zha, Wei, \& Liang, 2017; He, He, Xu, \& Shi, 2019). Accordingly, more and more companies would consider their environmental impacts while making operational and strategic decisions to meet social environmental protection standards. Yang and Chen (2018) state that many companies do volunteer to report their products and services' carbon footprints and endeavor to decrease carbon emissions. For instance, Swiss Post, HP, Sony and Vanke have taken part in the WWF Climate Savers Program and promise to reduce carbon emissions ${ }^{1}$.

So far, most studies on low-carbon supply chains have investigated the issue of how to strike a balance between financial benefit and the cost of environmental protection for physical goods (Bai, Chen, \& Xu, 2017; Zhang, Wang, Yu, \& Ren, 2019; Zhou \& Ye, 2018; Zu, Chen, \& Fan, 2018). By contrast, research on low-carbon service supply chains has been limited. Actually, service industry has become a major driving force for many countries' economic growth with the continuous

\footnotetext{
${ }^{1} \mathrm{https} / / /$ climatesavers.org/partners-companies/.
} 
improvement of people's living standard. However, some researchers argue that service industries such as transportation and tourism, have generated a large number of used products (e.g., waste cartons and plastic bags) to be disposable (He, He, \& Xu, 2018; Liu, Xu, \& Kouhpaenejad, 2013; Liu, Wang, Zhu, Wang, \& Shen, 2017). These would generate a huge amount of carbon emission, especially in the current rapid development of e-commerce era. According to the report by Guizhou Expressway Bureau, during the China National Day holiday, garbage generated by consumers' travel consumption was more than 1252 tons, which resulted in a great amount of carbon emission ${ }^{1}$. Therefore, it is of practical significance and essential for the governments and enterprises to control carbon emission in service supply chains.

When it comes to firms' sustainable operation, corporate social responsibility (CSR) is one of the most important factors that could affect enterprises' pricing and/or service decisions (Cruz \& Matsypura, 2009; Hsueh \& Chang, 2008). CSR here means companies voluntarily undertake the responsibility for the negative impacts of their business activities on the society, consumers and environment (Andersen \& Skjoett-Larsen, 2009). Some scholars have confirmed that CSR could significantly influence consumer satisfaction, consumer-firm identification, and positive attitudes towards enterprises (Eisingerich, Rubera, Seifert, \& Bhardwaj, 2011; Yoon, Gürhan-Canli, \& Schwarz, 2006). Nowadays, some companies (e.g., Travelocity, Airbnb, WalMart, and Ctrip) are increasingly incorporating the CSR into their strategic decision-making processes to improve their brand images. Additionally, government environmental regulations (e.g., Extended Producer Responsibility, Carbon tax etc.) and consumers' pressures also urge service enterprises to consider the negative environmental impact of their business activities. Hence, it is urgent to strengthen the research on how CSR affects service supply chains'operational performance.

Cooperation is one of the most effective means to improve enterprises' or a supply chain's operational performance (Cachon \& Lariviere, 2005; Luo, Chen, \& Wang, 2016; Wang et al., 2016; $\mathrm{Xu}, \mathrm{He}, \mathrm{Xu}, \&$ Zhang, 2017). Many scholars have proposed various coordination contracts, such as

1 http://hotel.chinairn.com/news/20170207/151737920.html. 
quantity discount, quantity flexibility, revenue sharing, cost-sharing and two-part tariff contracts, to help managers make better cooperative decisions and achieve Pareto improvement (Cachon \& Lariviere, 2005; Tsay, 1999; Zhou, Bao, Chen, \& Xu, 2016). Wherein, cost-sharing contracts have been proved to be effective and widely used to coordinate supply chains, especially when extra cost occurs (Ghosh \& Shah, 2015; Zhou, Guo, \& Zhou, 2018). Particularly, Zhou et al. (2016) propose and compare the cooperative advertising cost-sharing contract and the cooperative advertising and emission reduction cost-sharing contract. They revealed that the first contract cannot realize channel coordination but the second contract can under certain condition. Building on the previous research, we focus on exploring how cost-sharing contracts work in a low-carbon service SC. Differently, our paper incorporates firms' CSR behaviors and service decisions. Besides, many studies have confirmed that the differential game is more appropriate to characterize the emission reduction process (Zhou \& Ye, 2018; Zu et al., 2018). Hence, considering long-term cumulative carbon emission reduction is another main difference that this paper has.

Inspired by the real business problems, this study considers a low-carbon service SC consisting of a service provider (SP) who is accountable for emission reduction and service and a service integrator (SI) who is responsible for advertising. Under such a context, SP shares SI's advertising cost while SI has different types of costs (i.e., SP's service cost or emission reduction cost) to opt. Many scholars have indicated that upstream firm sharing the downstream firm's advertising cost outperforms the case of no cost sharing (Zhou et al., 2016). Accordingly, we here concentrate on the case in which SP always shares SI's advertising cost and omit the no sharing one, which will be discussed in Proposition 3. Consequently, given that SP shares the advertising cost, we propose three different cost-sharing contracts based on SI's distinct cost-sharing decisions, namely, (1) SI does not share any cost of SP (contract PA); (2) SI shares SP's carbon emission reduction cost (contract PAIE); and (3) SI shares SP's service cost (contract PAIS). The main purpose of this paper is to discuss the advantage of each contract in the presence of firms' CSR behaviors. Specifically, the research questions are: 
- What are the optimal decisions for chain members under different cost-sharing contracts?

- Which contract is the best option for each member, the service SC or the environment?

- How do the critical system factors, especially the CSR-related factors, affect the optimal outcomes and contract decisions?

To address the above questions, this article develops three SP-Stackelberg differential game models to investigate SP's service and emission reduction decisions as well as SI's advertising decision in the presence of CSR. Based on the proposed cost-sharing contracts, we derive the optimal service levels, carbon emission reduction and advertising efforts for the participants as well as their optimum cost-sharing rates. We further compare these three contracts to discuss the optimal contract choice from different perspectives. We also examine the impacts of CSR-related factors on SP's and SI's optimal outcomes. Through numerical examples and sensitivity analyses, we further check the roles some system parameters play in the low-carbon service SC.

We organize the remaining of this paper as follows. We briefly present the relevant literature in Section 2. In Section 3, the general differential game framework and assumptions are presented. Section 4 derives the equilibrium solutions and explores the impacts of environment-related factors. In Section 5, the comparative analyses among different contracts are conducted. Numerical experiments and sensitivity analyses are performed to validate and enrich our analytical results in Section 6. Finally, Section 7 summarizes the paper and provides several future research directions.

\section{Literature review}

Three main streams of literature are closely related to our work: (1) emission reduction in low-carbon supply chains; (2) corporate social responsibility; (3) cost-sharing contract.

\subsection{Emission reduction in low-carbon supply chains}

With the rapid increase of consumers' environmental awareness, more and more firms are spontaneously implementing low-carbon operations to address the social and civil environmental concerns so as to strengthen their brand image and expand their market shares (Cao \& Yu, 2018; 
Ghosh \& Shah 2015; Shen, Ding, Chen, Chan, Govindan, \& Wagner, 2017; Zhou et al., 2016; Zu et al., 2018). To promote sustainable development, many scholars and practitioners have paid much attention to firms' low-carbon activities (e.g., Bazan et al., 2016; Zu et al., 2018). Particularly, Luo et al. (2016) look into the effect of coopetition between two manufacturers who have different emission reduction efficiencies. They discover that manufacturers' coopetition behavior can result in more profits and less total carbon emissions. Ji, Zhang, and Yang (2017) consider a channel selection problem under both cap-and-trade regulation and consumers' environmental preference. They show that manufacturer can obtain benefit through adding an online channel when consumers' environmental preference is sufficiently high and the retailer is more favorable to the single-channel structure as consumers' environmental preference increases. Cao and Yu (2018) consider a capital-constrained and emission-dependent SC under carbon cap-and-trade mechanism. They uncover that carbon emission cap cannot affect the optimal ordering quantity under centralized decision. Yang et al. (2018) study a joint decision issue of manufacturer's channel selection and carbon emission reduction effort. Their findings indicate that products' features and the degree of consumers' channel preference exert significant influences on manufacturer's channel decision. Zhou and Ye (2018) develop a differential game model to investigate the joint emission reduction and coordination issues in a dual-channel low-carbon SC. Their results show that exogenous wholesale price and customers' channel preference towards offline channel play critical roles in setting emission reduction and advertising efforts.

Most of the previous studies focus on exploring the optimal emission abatement investment and/or SC coordination without considering firms' CSR behaviors or service decisions, which is one main feature that this paper differs from them.

\subsection{Corporate social responsibility}

The second stream of research relevant to our work is Corporate Social Responsibility (CSR). Numerous empirical studies have shown that CSR plays a crucial role in market expansion and 
brand improvement (see Andersen \& Skjoett-Larsen, 2009; Eisingerich et al., 2011; Lee \& Tang, 2017; Luo et al., 2016; Panda, Modak, \& Cardenas-Barron, 2017). Lombart and Louis (2014) explore the effect of retailer's CSR behavior by collecting data from 352 consumers of a French grocery retailer. They discover that perceived CSR is positively (negatively) associated with the retailer personal conscientiousness (disingenuousness). Han and Yoon (2015) integrate environmental awareness, perceived effectiveness, reputation and eco-friendly behavior to examine tourists' intention formation when choosing an environmentally responsible hotel. They confirm that higher tourists' environmental desire and firms' environmentally responsible reputation could enhance guests' pro-environmental intention. Agan, Kuzey, Acar, and Acikgoz (2016) develop a partial least square structural equation model to examine the relationship between CSR and environmental supplier development. Their finding demonstrates that CSR is positively associated with environmental supplier development. Some researchers have discussed the impacts of CSR from a theoretical point of view (e.g., Hsueh, 2014; Letizia \& Hendrikse, 2016; Yuen, Thai, Wong, \& Wang, 2018). Particularly, Arya and Mittendorf (2015) discuss the effects of government's subsidies for CSR on SC members' production and donation decisions. They underscore that downstream firms, who engage in CSR activities, become more sensitive to supplier's pricing decisions and government's subsidy can incentivize firms to donate. Banerjee and Wathieu (2017) investigate the optimal CSR effort decisions in the monopoly and duopoly market. They find that CSR has a substitute for product quality to some extent. Wu, Li, Gou, and Gu (2017) consider a two-level international SC where the oversea supplier's social misconduct may damage original equipment manufacturer's CSR towards consumers. Panda et al. (2017) investigate how CSR affects chain members' profits and employ the revenue sharing contract to coordinate the socially responsible closed-loop SC. They indicate that CSR is a costly endeavor but it can generate an access for the manufacturer to adjust surplus profit share.

Most aforementioned studies assume that only one of chain members has CSR behavior and all of them consider a static context. Diverging from them, the focus of this research lies in developing 
differential game models to investigate the issue of how service firms' CSR behaviors affect their joint curbing carbon emission and contract designs.

\subsection{Cost-sharing contract}

The last stream of literature related to our paper is regarding cost-sharing contract, which has a long-standing tradition in SC coordination management (e.g., Chutani \& Sethi, 2018; Frisk, Göthe-Lundgren, Jörnsten, \& Rönnqvistab, 2010; Liu, Quan, Li, \& Forrest, 2018; Wang et al., 2016). Ghosh and Shah (2015) investigate the influence of cost-sharing contract on chain members' operational decisions in a green supply chain. They develop and compare the retailer providing cost-sharing contract and both manufacturer and retailer bargaining on the contract. Xu et al. (2017) employ the wholesale price contract and cost-sharing contract to coordinate the green supply chain under cap-and-trade regulation. They show that both contracts can achieve SC coordination. Yang, Shan, and Jin (2017) investigate the capacity planning issue by incorporating full capacity cost-sharing contract and partial capacity cost-sharing contract. Comparing these two contracts yields that the retailer would share more cost but less capacity quantity in the second contract than that in the first one. Bai et al. (2017) look into the SC coordination issue in a sustainable SC system with deteriorating items. They develop a two-part tariff contract and a revenue and promotional cost-sharing contract in order to realize SC coordination. They show that the former contract is more robust than the latter. Yang and Chen (2018) examine the impacts of retailer's revenue-sharing contract and cost-sharing contract towards the manufacturer in a retailer-driven carbon emission reduction SC, considering consumers' environmental awareness and carbon tax. They confirm that both contracts can contribute to the performance improvement and can stimulate the manufacturer to enhance emission abatement effort. Considering the problem of free riding in a dual-channel SC, Zhou et al. (2018) investigate the pricing decision of a manufacturer and propose a service-cost sharing contract to improve the SC's performance.

The above research results have consistently indicated that cost-sharing contract is an effective 
coordination mechanism. The novelty of this research is that we focus on exploring the joint decisions of optimal service level, carbon abatement and advertising efforts and analyze the advantage of each cost-sharing contract under different circumstances by incorporating firms' CSR behaviors.

\section{Model description and assumptions}

Table 1 Notation and definitions.

\begin{tabular}{|c|c|}
\hline Notation & Definitions \\
\hline$S(t)$ & Effort towards service of SP \\
\hline$E(t)$ & Effort towards emission reduction of SP \\
\hline$A(t)$ & Effort towards advertising of SI \\
\hline$\psi(t)$ & SP's cost-sharing rate on SI's advertising effort, $0 \leq \psi(t) \leq 1$ \\
\hline$\phi(t)$ & SI's cost-sharing rate on SP's basic service or emission reduction effort, $0 \leq \phi(t) \leq 1$ \\
\hline$G(t)$ & $\begin{array}{l}\text { Reduction of emissions at time } t \in[0,+\infty) \text { with initial carbon emission reduction } \\
\left.G(t)\right|_{t=0}=G_{0} \geq 0\end{array}$ \\
\hline$R_{i}$ & Marginal profit of SP or SI, $i=S P, S I$ \\
\hline$\mu_{a}, \mu_{e}$ & $\begin{array}{l}\text { Coefficient associated with SP's emission reduction effort and SI's advertising effort in the } \\
\text { function of emission reduction, } \mu_{a}>0, \mu_{e}>0 \\
\text { Decay rate of the emission reduction }\end{array}$ \\
\hline$D(t)$ & Demand for the service product at time $t$ \\
\hline$\alpha, \beta, \gamma$ & $\begin{array}{l}\text { Coefficient associated with SP's service effort, SI's advertising effort and the emission } \\
\text { reduction in the demand function, } \alpha>0, \beta>0, \gamma>0\end{array}$ \\
\hline$k_{j}$ & $\begin{array}{l}\text { Cost sensitivity coefficient to SP's service and emission reduction efforts, and SI's } \\
\text { advertising effort, } j=s, e, a\end{array}$ \\
\hline$\theta$ & Corporate social responsibility of the supply chain \\
\hline$\lambda$ & Relative CSR of SI relative to SP, $1-\lambda$ denotes SP's relative CSR \\
\hline$U_{i}^{l}(t)$ & Utility function of SP, SI or supply chain in $l$ contract, $i=S P, S I, S C ; l=P A, P A I E, P A I S$ \\
\hline$V_{i}^{l}(t)$ & Net value function of SP or SI, $i=S P, S I ; l=P A, P A I E, P A I S$ \\
\hline$\rho$ & Discount rate \\
\hline
\end{tabular}

Consider a two-echelon service supply chain consisting of an upstream service provider (SP) and a downstream service integrator (SI), both of whom have certain CSR preferences. To cooperatively reduce carbon emission and improve their social brand image, SP is responsible for emission reduction and service level and SI is responsible for low-carbon advertising. As discussed in Introduction, we suppose that SP provides an advertising cost-sharing contract to incentivize SI (Balachandran \& Radhakrishnan, 2005). Under this context, it is supposed that SI may have three cost-sharing decisions: (1) not sharing any effort cost of SP (contract PA), namely, only SP shares 
SI's advertising cost; (2) sharing the emission reduction cost of SP (contract PAIE), that is, SP shares SI's advertising cost and SI shares SP's carbon emission reduction cost, respectively; (3) sharing the service cost of SP (contract PAIS). In contrast to contract PAIE, SI directly shares SP's service cost. Notice that contract PA would be called as "one-way contract" and contract PAIE or PAIS would be called as "two-way contract" in the main text. Following many previous papers (Xia et al., 2018; Zhang, Lei, Zhang, \& Song, 2017), we also assume that the information is symmetric for every participant. Table 1 summarizes the main notation and its definitions used in this paper.

The dynamic of emission reduction could be governed by a differential equation, which is jointly affected by SP's emission reduction and SI's advertising efforts (Zhou \& Ye, 2018). It is intuitive that SP's emission reduction effort can directly enhance the accumulation of emission reduction. The low-carbon advertising would also improve consumers' environmental awareness, which may contribute to carbon emission reduction indirectly. For example, tourists can control their own littering behaviors while traveling, thus reducing the amount of carbon emission. If there are no efforts, the emission reduction level will decay over time (Jorgensen, Taboubi, \& Zaccour, 2001). Consequently, the differential equation of emission reduction can be formulated as

$$
\left\{\begin{array}{c}
\dot{G}(t)=\mu_{e} E(t)+\mu_{a} A(t)-\mu_{g} G(t) \\
G(0)=G_{0} \geq 0
\end{array}\right.
$$

where $G(t)$ represents the emission reduction at time $t$, and $G_{0}$ is the initial emission reduction amount. In addition, $E(t)$ and $A(t)$ refer to SP's emission reduction effort and SI's advertising effort at time $t$, respectively. Parameters $\mu_{e}$ and $\mu_{a}$ are the sensitivity coefficients towards emission reduction and advertising efforts. Parameter $\mu_{g}$ represents the decay rate of emission reduction over time.

Following prior studies (He, Prasad, \& Sethi, 2009; Zhang, Gou, Liang, \& Huang, 2013; Zhang, Hafezi, Zhao, \& Shi, 2017), we assume that the chain members are price-takers ${ }^{1}$. Namely, the retail price is constant under the long-term market equilibrium. However, we assume that SP can

\footnotetext{
${ }^{1}$ This assumption is more consistent with the service industry. For example, the ticket prices of most scenic spots and/or the retail prices of restaurant dishes almost keep constant.
} 
adjust the service level whenever necessary. For example, tour attractions and/or restaurants may employ holiday part-time workers in response to temporary demand change. Therefore, the demand for the service products can be written as $D(S(t), E(t), G(t), p)=d_{0}-b p+\alpha S(t)+\beta A(t)+$ $\gamma G(t)$, where $d_{0}$ denotes basic demand, $p$ denotes long-term market equilibrium retail price and $b$ represents price sensitive coefficient. Without loss of generality, we define $D_{0} \equiv d_{0}-b p$ and further assume $D_{0}=0$ to simplify our models since it has no significant effect on our main results (Chutani \& Sethi, 2018). We hereafter use notation $R_{i}, i=S P, S I$ to represent the marginal profit of SP or SI. As a consequence, the demand function is degenerated to the following expression.

$$
D(S(t), A(t), G(t))=\alpha S(t)+\beta A(t)+\gamma G(t)
$$

where coefficients $\alpha, \beta$ and $\gamma$ denote the demand sensitivity coefficient towards SP's service effort, SI's advertising effort and emission reduction level, respectively. Notice that $\gamma$ can be also regarded as consumers' low-carbon preference.

In consistent with some previous papers (Jorgensen et al., 2001; Zhang et al., 2013), we utilize the quadratic functions to measure the effort costs. The efforts cost towards service, emission reduction and advertising at time $t$ can be expressed as:

$$
\left\{\begin{array}{l}
C_{S}(S(t))=\frac{k_{S} S^{2}(t)}{2} \\
C_{E}(E(t))=\frac{k_{e} E^{2}(t)}{2} \\
C_{A}(A(t))=\frac{k_{a} A^{2}(t)}{2}
\end{array}\right.
$$

where $k_{j}(j=s, e, a)$ represents cost sensitivity coefficient towards SP's service effort and emission reduction effort, as well as SI's advertising effort, respectively.

Due to the focus of this paper is on environmental protection, we assume that only the effect of firms' CSR behaviors in the form of environment concern is embodied in our models. We employ the notation $\theta \in[0,1]$ to represent the fraction of the whole supply chain' CSR relative to the profitability. Following the work of Panda and Modak (2016), we also utilize the proportion $\lambda \in[0,1]$ to denote SI's relative CSR preference compared to SP (hereafter relative CSR) and the 
rest $1-\lambda$ denotes SP's relative CSR preference. When $0 \leq \lambda<\frac{1}{2}$, it means SI has a lower CSR than SP. The opposite is right for SP. $\lambda=\frac{1}{2}$ means no CSR difference between the two service companies.

Therefore, the utility functions of upstream SP and downstream SI satisfy the following differential equations when there is no cost-sharing contract between SP and SI.

$$
\begin{gathered}
U_{S P}=\int_{0}^{\infty} e^{-\rho t}\left\{R_{S P} D[s(t), A(t), G(t)]+(1-\lambda) \theta G(t)-\frac{k_{S} S^{2}(t)}{2}-\frac{k_{e} E^{2}(t)}{2}\right\} d t \\
U_{S I}=\int_{0}^{\infty} e^{-\rho t}\left\{R_{S I} D[S(t), A(t), G(t)]+\lambda \theta G(t)-\frac{k_{a} A^{2}(t)}{2}\right\} d t
\end{gathered}
$$

where $\rho$ is the common discount rate of SC members' utilities over time. The first terms of $U_{S P}$ and $U_{S I}$ denote sale revenues; the second terms represent CSR utilities of SP and SI; the last terms are SP's and SI's corresponding effort costs.

\section{Equilibrium solutions and analysis}

In this section, we develop three cost-sharing differential game models and attempt to derive the analytical solutions. Furthermore, we analyze the influences of environmental factors on equilibrium solutions. Since the game is played over an infinite time horizon, it is reasonable to seek a steady state feedback Stackelberg equilibrium. Many previous scholars have argued that this feedback control strategy is more meaningful than the open-loop control strategy (Cellini \& Lambertini, 2004; Piga, 2000).

\subsection{Equilibrium solutions under contract PA (Model PA)}

In this configuration, only SP provides an advertising cost-sharing rate for SI. The game sequence is as follows: SP first decides her cost-sharing rate, and then announces the service level and emission reduction effort. Afterward, SI determines his advertising effort. According to Eqs. (4) and (5), the optimization problems of SP and SI are standard optimal control problems given by: 


$$
\begin{aligned}
\max _{S, E, \varphi} U_{S P}^{P A}= & \int_{0}^{\infty} e^{-\rho t}\left\{R_{S P} D[S(t), A(t), G(t)]+(1-\lambda) \theta G(t)-\frac{k_{S} S^{2}(t)}{2}-\frac{k_{e} E^{2}(t)}{2}\right. \\
& \left.-\frac{\varphi(t) k_{a} A^{2}(t)}{2}\right\} d t \\
\max _{A} U_{S I}^{P A}= & \int_{0}^{\infty} e^{-\rho t}\left\{R_{S I} D[S(t), A(t), G(t)]+\lambda \theta G(t)-\frac{(1-\varphi(t)) k_{a} A^{2}(t)}{2}\right\} d t
\end{aligned}
$$

both are subject to

$$
\left\{\begin{array}{c}
D(S(t), A(t), G(t))=\alpha S(t)+\beta A(t)+\gamma G(t) \\
\dot{G}(t)=\mu_{a} A(t)+\mu_{e} E(t)-\mu_{g} G(t) \\
G(0)=G_{0} \geq 0
\end{array}\right.
$$

For simplicity and clarity of writing, we hereafter omit time factor when no confusion arises. We henceforth employ notation $V_{S P}^{l}\left(V_{S I}^{l}\right)$ to represent the net utility of the SP (SI) after time $t$ in the Hamilton-Jacobi-Bellman equation (HJB). In Model PA, $V_{S P}^{P A}$ and $V_{S I}^{P A}$ should meet the following equations:

$$
\begin{aligned}
& \rho V_{S P}^{P A}=\max _{S, E, \varphi \geq 0}\left[R_{S P}(\alpha S+\beta A+\gamma G)+(1-\lambda) \theta G-\frac{k_{S} S^{2}}{2}-\frac{k_{e} E^{2}}{2}-\frac{\varphi k_{a} A^{2}}{2}+V_{S P}^{P A^{\prime}}\left(\mu_{a} A\right.\right. \\
& \left.\left.+\mu_{e} E-\mu_{g} G\right)\right] \\
& \rho V_{S I}^{P A}=\max _{A \geq 0}\left[R_{S I}(\alpha S+\beta A+\gamma G)+\lambda \theta G-\frac{(1-\varphi) k_{a} A^{2}}{2}+V_{S I}^{P A \prime}\left(\mu_{a} A+\mu_{e} E-\mu_{g} G\right)\right]
\end{aligned}
$$

We solve these problems by backward induction. Taking derivatives of the HJB equations with respect to $S, E, A$ and $\varphi$, we can obtain the optimal responsive service level, emission reduction and advertising efforts as well as the participation rate, which are expressed as

$$
\left\{\begin{array}{c}
S=\frac{\alpha R_{S P}}{k_{S}} \\
A=\frac{\beta R_{S I}+\mu_{a} V_{S I}^{P A \prime}}{(1-\varphi) k_{a}} \\
E=\frac{\mu_{e} V_{S P}^{P A^{\prime}}}{k_{e}} \\
\varphi=\frac{\beta\left(2 R_{S P}-R_{S I}\right)+\mu_{a}\left(2 V_{S P}^{P A \prime}-V_{S I}^{P A \prime}\right)}{\beta\left(2 R_{S P}+R_{S I}\right)+\mu_{a}\left(2 V_{S P}^{P A^{\prime}}+V_{S I}^{P A \prime}\right)}
\end{array}\right.
$$


By inserting the above Eq. (11) into Eqs. (9) and (10), we can find that the optimal function relying on $G$ is the solution of the HJB equation. According to Eqs. (9)-(11), we can set the general forms of these functions as linear expressions: $V_{S P}^{P A}=a_{1} G+b_{1}$ and $V_{S I}^{P A}=c_{1} G+d_{1}$, where $a_{1}$, $b_{1}, c_{1}$ and $d_{1}$ are the constants to be solved. Differentiating the general functions, it leads to $V_{S P}^{P A \prime}=a_{1}$ and $V_{S I}^{P A \prime}=c_{1}$. We can further obtain the optimal net utilities of SP and SI shown as below.

$$
\begin{aligned}
V_{S P}^{P A *} & =a_{1} G+b_{1}=\left[\theta(1-\lambda)+\gamma R_{S P}-a_{1} \mu_{g}\right] G \\
& +\frac{4 \alpha^{2} k_{a} k_{e} R_{S P}^{2}+k_{e} k_{S}\left[2 \beta R_{S P}+\beta R_{S I}+\left(2 a_{1}+c_{1}\right) \mu_{a}\right]^{2}+4 a_{1}^{2} k_{a} k_{s} \mu_{e}^{2}}{8 k_{a} k_{e} k_{S}} \\
V_{S I}^{P A *} & =c_{1} G+d_{1}=\left(\theta \lambda+\gamma R_{S I}-c_{1} \mu_{g}\right) G+\frac{4 \alpha^{2} k_{a} k_{e} R_{S P} R_{S I}}{4 k_{a} k_{e} k_{S}} \\
& +\frac{k_{e} k_{S}\left(\beta R_{S I}+c_{1} \mu_{a}\right)\left[2 \beta R_{S P}+\beta R_{S I}+\left(2 a_{1}+c_{1}\right) \mu_{a}\right]+4 a_{1} c_{1} k_{a} k_{S} \mu_{e}^{2}}{4 k_{a} k_{e} k_{S}}
\end{aligned}
$$

Solving the above linear equations with respect to $a_{1}, b_{1}, c_{1}$ and $d_{1}$, we can obtain the optimum feedback equilibrium solutions of Model PA shown in Lemma 1. All solving processes are provided in Appendix A.

Lemma 1. In Model PA, the feedback equilibrium solutions are

$$
\left\{\begin{array}{c}
S^{P A *}=\frac{\alpha R_{S P}}{k_{S}} \\
A^{P A *}=\frac{\theta(2-\lambda) \mu_{a}+\left[\gamma \mu_{a}+\beta\left(\rho+\mu_{g}\right)\right]\left(2 R_{S P}+R_{S I}\right)}{2 k_{a}\left(\rho+\mu_{g}\right)} \\
E^{P A *}=\frac{\mu_{e}\left[(1-\lambda) \theta+\gamma R_{S P}\right]}{k_{e}\left(\rho+\mu_{g}\right)} \\
\varphi^{P A *}=\frac{\theta(2-3 \lambda) \mu_{a}+\left(2 R_{S P}-R_{S I}\right)\left[\gamma \mu_{a}+\beta\left(\rho+\mu_{g}\right)\right]}{\theta(2-\lambda) \mu_{a}+\left(2 R_{S P}+R_{S I}\right)\left[\gamma \mu_{a}+\beta\left(\rho+\mu_{g}\right)\right]}
\end{array}\right.
$$

Substituting the optimal solutions Eq. (14) into Eq. (1) yields the following equation:

$$
\dot{G}(t)^{P A *}=\frac{\mu_{a}\left[\beta\left(\rho+\mu_{g}\right) R_{S I}+\mu_{a}\left(\lambda \theta+\gamma R_{S P}\right)\right]}{\left(1-\varphi^{P A *}\right)\left(\rho+\mu_{g}\right) k_{a}}+\frac{\mu_{e}^{2}\left[(1-\lambda) \theta+\gamma R_{S P}\right]}{k_{e}\left(\rho+\mu_{g}\right)}-\mu_{g} G(t)^{P A *}
$$

According to the dynamic function of emission reduction equation Eq. (15), we can obtain the time trajectory of accumulated emission reduction as follows: 


$$
G(t)^{P A *}=G_{\infty}^{P A *}+\left(G_{0}-G_{\infty}^{P A *}\right) e^{-\mu_{g} t}
$$

where $G_{0}=G(0)$ and $G_{\infty}^{P A *}$ refers to the steady-state emission reduction, which can be given as $G_{\infty}^{P A *}=\left(\mu_{e} / \mu_{g}\right) E^{P A *}+\left(\mu_{a} / \mu_{g}\right) A^{P A *}$. The specific expression of $G_{\infty}^{P A *}$ is shown in Eq. (17).

$$
G_{\infty}^{P A *}=\frac{2 k_{a}\left[\theta(1-\lambda)+\gamma R_{S P}\right] \mu_{e}^{2}+k_{e} \mu_{a}\left\{\theta(2-\lambda) \mu_{a}+2 R_{S P}\left[\gamma \mu_{a}+\beta\left(\rho+\mu_{g}\right)\right]+R_{S I}\left[\gamma \mu_{a}+\beta\left(\rho+\mu_{g}\right)\right]\right\}}{2 \gamma k_{a} k_{e}\left(\rho+\mu_{g}\right)}
$$

Then, substituting the above equilibriums and state equation into the objective functions of SP and SI, we can obtain their optimal utilities under contract PA shown in Eqs. (18)-(19). To facilitate writing, we hereafter define: $M_{1} \equiv \theta(1-\lambda)+\gamma R_{S P} ; M_{2} \equiv \theta(2-\lambda) \mu_{a}+\left(2 R_{S P}+R_{S I}\right)\left[\gamma \mu_{a}+\right.$ $\left.\beta\left(\rho+\mu_{g}\right)\right] ; M_{3} \equiv \theta(1+\lambda)+\gamma R_{m}+2 \gamma R_{r} ; M_{4} \equiv(2+\gamma) \theta \lambda \mu_{a}+\gamma R_{r}\left[(2+\gamma) \mu_{a}+\beta\left(\rho+\mu_{g}\right)\right]$ $M_{5} \equiv 2 \gamma R_{m}\left[\theta(\gamma+2 \lambda+\gamma \lambda)+2 \gamma(1+\gamma) R_{r}\right] \quad ; \quad M_{6} \equiv \theta^{2}(1+\lambda)(\gamma+4 \lambda+\gamma \lambda)+\gamma^{3} R_{m}^{2}+$ $4 \gamma \theta(1+\gamma+3 \lambda+\gamma \lambda) R_{r}+4 \gamma^{2}(2+\gamma) R_{r}^{2}$

$$
\begin{gathered}
U_{S P}^{P A *}=\frac{e^{-\rho t}\left\{\left[4 M_{1}\left(2 k_{a} M_{1} \mu_{e}^{2}+k_{e} \mu_{a} M_{2}\right)\right] / \gamma+\left\{4 k_{a}\left[k_{s} M_{1}^{2} \mu_{e}^{2}+\alpha^{2} k_{e} R_{m}^{2}\left(\rho+\mu_{g}\right)^{2}\right]+k_{e} k_{s} M_{2}^{2}\right\} / k_{s}\right\}}{8 k_{a} k_{e}\left(\rho+\mu_{g}\right)^{2}} \\
U_{S I}^{P A *}=\frac{e^{-\rho t}\left\{4 k_{a}\left\{(1+\gamma) k_{s} M_{1}\left(\theta \lambda+\gamma R_{r}\right) \mu_{e}^{2}+\alpha^{2} \gamma k_{e} R_{m} R_{r}\left(\rho+\mu_{g}\right)^{2}\right\}+k_{e} k_{s} M_{2} M_{4}\right\}}{4 \gamma k_{a} k_{e} k_{S}\left(\rho+\mu_{g}\right)^{2}}
\end{gathered}
$$

According to Lemma 1, we can obtain the following Proposition 1 that demonstrates the effects of environmental factors (i.e., $\lambda, \theta$ and $\gamma$ ) on the equilibrium solutions.

Proposition 1. The optimal service level, emission reduction and advertising efforts, as well as SP'S cost-sharing rate hold the properties as below:

(i) $\partial S^{P A *} / \partial \theta=0 ; \partial S^{P A *} / \partial \lambda=0 ; \partial S^{P A *} / \partial \gamma=0$.

(ii) $\partial E^{P A *} / \partial \theta>0 ; \partial E^{P A *} / \partial \lambda<0 ; \partial E^{P A *} / \partial \gamma>0$.

(iii) $\partial A^{P A *} / \partial \theta>0 ; \partial A^{P A *} / \partial \lambda<0 ; \partial A^{P A *} / \partial \gamma>0$.

(iv) When $R_{S P} / R_{S I}<(1-\lambda) / \lambda, \partial \varphi^{P A *} / \partial \theta>0$ and $\partial \varphi^{P A *} / \partial \gamma<0$, otherwise $\partial \varphi^{P A *} / \partial \theta<0$ and $\partial \varphi^{P A *} / \partial \gamma>0 ; \partial \varphi^{P A *} / \partial \lambda<0$.

The first part of Proposition 1 indicates that SP decides her service level without considering CSR, SI's relative CSR and consumers' low-carbon preference. It can be explained that the service 
is independent of the parameters $\lambda, \theta$ and $\gamma$ and there is no service cost transfer between SP and SI in this setting. From Proposition 1(ii)-(iii), it is clear that the optimal carbon emission reduction and advertising efforts increase in supply chain's CSR $(\theta)$ and consumers' low-carbon preference $(\gamma)$. The reason is straightforward because with higher $\theta$ and $\gamma$, the chain members can attract more consumers. Contrarily, both emission reduction and advertising efforts decrease with relative CSR. A higher $\lambda$ means that SP has relatively low CSR, so SP is unlikely to participate in a low-carbon environmental activity. This leads to lower emission reduction effort, thereby reducing the efficiency of SI's advertising. As per Proposition 1(iv), it is easy to find that higher $\lambda$ will reduce the motivation of SP to share advertising cost. This further explains why SI will cut down his advertising investment as $\lambda$ increases. SP's double reduction effects would be more obvious than the utility increment of SI acquiring from CSR. Moreover, $R_{S P} / R_{S I}<(1-\lambda) / \lambda$ means SP can gain relatively high utility from CSR but relatively low marginal profit from sales in comparison to SI. Hence, when $\theta$ increases, the relative advantage of SP will rise. When $\gamma$ increases, it will result in higher demand so as to drive up the motivation of SI investing in advertising. This is the reason why SP would offer a lower participation rate with the increase of consumers' low-carbon preference. The opposite is true for SP when $R_{S P} / R_{S I}>(1-\lambda) / \lambda$.

\subsection{Equilibrium solutions under contract PAIE (Model PAIE)}

Under contract PAIE, the sequence of events can be described as follows: first of all, both SP and SI announce their cost-sharing rates. Then, SP decides her optimal service level and emission reduction effort. After that SI in response to SP's optimal reaction makes an advertising decision. The optimization problems of chain members can be given as follows.

$$
\begin{aligned}
\max _{S, E, \varphi} U_{S P}^{P A I E}= & \int_{0}^{\infty} e^{-\rho t}\left\{R_{S P} D[S(t), A(t), G(t)]+(1-\lambda) \theta G(t)-\frac{k_{S} S^{2}(t)}{2}\right. \\
& \left.-\frac{(1-\phi(t)) k_{e} E^{2}(t)}{2}-\frac{\varphi(t) k_{a} A^{2}(t)}{2}\right\} d t
\end{aligned}
$$




$$
\begin{aligned}
\max _{A, \phi} U_{S I}^{P A I E}= & \int_{0}^{\infty} e^{-\rho t}\left\{R_{S I} D[S(t), A(t), G(t)]+\lambda \theta G(t)-\frac{(1-\varphi(t)) k_{a} A^{2}(t)}{2}\right. \\
& \left.-\frac{\phi(t) k_{e} E^{2}(t)}{2}\right\} d t
\end{aligned}
$$

both are subject to Eq. (8).

Using similar notation, the optimal net utilities of SP and SI must satisfy the following HJB equations:

$$
\begin{aligned}
& \rho V_{S P}^{P A I E}=\max _{S, E, \varphi \geq 0}\left[R_{S P}(\alpha S+\beta A+\gamma G)+(1-\lambda) \theta G-\frac{k_{S} S^{2}}{2}-\frac{(1-\phi) k_{e} E^{2}}{2}-\frac{\varphi k_{a} A^{2}}{2}\right. \\
& \left.+V_{S P}^{P A I E^{\prime}}\left(\mu_{a} A+\mu_{e} E-\mu_{g} G\right)\right] \\
& \rho V_{S I}^{P A I E}=\max _{A, \phi \geq 0}\left[R_{S I}(\alpha S+\beta A+\gamma G)+\lambda \theta G-\frac{(1-\varphi) k_{a} A^{2}}{2}-\frac{\phi k_{e} E^{2}}{2}+V_{S I}^{P A I E^{\prime}}\left(\mu_{a} A+\mu_{e} E\right.\right. \\
& \left.\left.-\mu_{g} G\right)\right]
\end{aligned}
$$

We can obtain the service companies' time-consistent Sub-game optimal responsive decisions shown in Eq. (24) by solving the first-order conditions.

$$
\left\{\begin{array}{c}
A=\frac{\beta R_{S I}+\mu_{a} V_{S I}^{P A I E^{\prime}}}{(1-\varphi) k_{a}} \\
S=\frac{\alpha R_{S P}}{k_{S}} \\
E=\frac{\mu_{e} V_{S P}^{P A I E^{\prime}}}{(1-\phi) k_{e}} \\
\varphi=\frac{\beta\left(2 R_{S P}-R_{S I}\right)+\mu_{a}\left(2 V_{S P}^{P A I E^{\prime}}-V_{S I}^{P A I E^{\prime}}\right)}{\beta\left(2 R_{S P}+R_{S I}\right)+\mu_{a}\left(2 V_{S P}^{P A I E^{\prime}}+V_{S I}^{P A I E^{\prime}}\right)} \\
\phi=\frac{2 V_{S I}^{P A I E^{\prime}}-V_{S P}^{P A I E^{\prime}}}{2 V_{S I}^{P A I E^{\prime}}+V_{S P}^{P A I E \prime}}
\end{array}\right.
$$

By substituting the above Eq. (24) into Eqs. (22)-(23), we can get the stationary feedback Stackelberg equilibrium solutions shown in Lemma 2.

Lemma 2. In Model PAIE, the feedback equilibrium solutions are given by 


$$
\left\{\begin{array}{c}
S^{P A I E *}=\frac{\alpha R_{S P}}{k_{S}} \\
E^{P A I E *}=\frac{\left[\theta(1+\lambda)+\gamma R_{S P}+2 \gamma R_{r}\right] \mu_{e}}{2 k_{e}\left(\rho+\mu_{g}\right)} \\
A^{P A I E *}=\frac{\beta\left(\rho+\mu_{g}\right) R_{S I}+\mu_{a}\left(\lambda \theta+\gamma R_{S P}\right)}{\left(1-\varphi^{P A I E *}\right)\left(\rho+\mu_{g}\right) k_{a}} \\
\varphi^{P A I E *}=\frac{\theta(2-3 \lambda) \mu_{a}+\left(2 R_{S P}-R_{S I}\right)\left[\gamma \mu_{a}+\beta\left(\rho+\mu_{g}\right)\right]}{\theta(2-\lambda) \mu_{a}+\left(2 R_{S P}+R_{S I}\right)\left[\gamma \mu_{a}+\beta\left(\rho+\mu_{g}\right)\right]} \\
\phi^{P A I E *}=\frac{\gamma\left(2 R_{S I}-R_{S P}\right)-\theta(1-3 \lambda)}{\theta(1+\lambda)+\gamma\left(2 R_{S I}+R_{S P}\right)}
\end{array}\right.
$$

Substituting the above optimal feedback equilibrium solutions into the differential function of emission reduction equation Eq. (1), we can obtain $G(t)^{P A I E *}$ under contract PAIE as follows.

$$
\left\{\begin{array}{c}
G(t)^{P A I E *}=G_{\infty}^{P A I E *}+\left(G_{0}-G_{\infty}^{P A I E *}\right) e^{-\mu_{g} t} \\
G_{0}=G(0) \geq 0
\end{array}\right.
$$

Similarly, $G_{\infty}^{\text {PAIE* }}=\left(\mu_{e} / \mu_{g}\right) E^{\text {PAIE* }}+\left(\mu_{a} / \mu_{g}\right) A^{\text {PAIE* }}$. For the sake of readability, we omit the specific expression and transfer it into Appendix A. We can further have their corresponding optimal utilities shown as below:

$$
\begin{gathered}
U_{S P}^{P A I E *}=\frac{e^{-\rho t}\left\{\left[4 M_{1}\left(k_{a} M_{3} \mu_{e}^{2}+k_{e} \mu_{a} M_{2}\right)\right] / \gamma+\left\{2 k_{a}\left[k_{s} M_{1} M_{3} \mu_{e}^{2}+2 \alpha^{2} k_{e} R_{m}^{2}\left(\rho+\mu_{g}\right)^{2}\right]+k_{e} k_{S} M_{2}^{2}\right\} / k_{s}\right\}}{8 k_{a} k_{e}\left(\rho+\mu_{g}\right)^{2}} \\
U_{S I}^{P A I E *}=\frac{e^{-\rho t}\left\{k_{a}\left[k_{s} \mu_{e}^{2}\left(M_{5}+M_{6}\right)+8 \alpha^{2} \gamma k_{e} R_{m} R_{r}\left(\rho+\mu_{g}\right)^{2}\right]+2 k_{e} k_{S} M_{2} M_{4}\right\}}{8 \gamma k_{a} k_{e} k_{S}\left(\rho+\mu_{g}\right)^{2}}
\end{gathered}
$$

The following Proposition 2 characterizes the impacts of supply chain members' equilibrium solutions with respect to $\theta, \lambda$ and $\gamma$. Because some properties in this model are similar to that in Model PA, we here just present some different results.

Proposition 2. The optimal emission reduction efforts and SI's cost-sharing rate have the following properties:

(i) $\partial E^{P A I E *} / \partial \theta>0 ; \partial E^{P A I E *} / \partial \lambda>0 ; \partial E^{P A I E *} / \partial \gamma>0$.

(ii) When $R_{S P} / R_{S I}>(1-\lambda) / \lambda$, $\partial \phi^{P A I E *} / \partial \theta>0$ and $\partial \phi^{P A I E *} / \partial \gamma<0$, otherwise $\partial \phi^{\text {PAIE* }} /$ $\partial \theta<0$ and $\partial \phi^{P A I E *} / \partial \gamma>0 ; \partial \phi^{P A I E *} / \partial \lambda>0$.

According to Proposition 2(i), SP's optimal carbon emission reduction effort increases in CSR, 
relative CSR and consumers' low-carbon preference. Furthermore, we can conclude that SI's emission reduction cost-sharing contract can transfer his CSR preference to his partner so that the SP would like to implement higher emission reduction effort as $\lambda$ increases, thereby enhancing the environmental friendliness of the entire service SC. In this way, contract PAIE indeed can promote the cooperation between two members. Moreover, Proposition 2(ii) demonstrates that when SI can get more utilities from implementing low-carbon program than the revenue of selling products, he will provide higher cost-sharing rate for SP with the increase of $\theta$ or the decrease of $\gamma$. It is also evident that higher relative CSR would motivate SI to offer higher cost-sharing rate.

Proposition 3. In Model PAIE, the cost-sharing contract decisions of SP and SI are presented in Table 2, where $\bar{\theta}_{1}=\frac{\left(2 R_{S P}-R_{S I}\right)\left[\gamma \mu_{a}+\beta\left(\rho+\mu_{g}\right)\right]}{(3 \lambda-2) \mu_{a}}$ and $\bar{\theta}_{2}=\frac{\gamma\left(2 R_{S I}-R_{S P}\right)}{(1-3 \lambda)}$.

We employ Fig. 1 to facilitate our discussions. Proposition 3 demonstrates that CSR, relative CSR and marginal profits of chain members strongly affect SP's and SI's contract designs. In general, when the joint effect of CSR and relative CSR is relatively low, SP would like to provide an advertising cost-sharing contract but SI always does not offer any contract (region 1: Fig. 1(LR1), (MR1), and (HR1)); when it is moderate, both SP and SI would implement cost-sharing contracts (region 2: Fig. 1(LR2), (MR2) and (HR2)). However, when it is relatively high, the contract decisions of SP and SI are opposite to that in a lower situation (region 3: Fig. 1(LR3), (MR3) and (HR3)). From the perspective of marginal profit, when SI has a sufficiently low marginal profit, region 3 is the smallest (see LR3). That means it will motivate SP to provide a positive participation rate while SI is getting less desire to implement a cost-sharing contract. When there is no too large difference between SP's and SI's marginal profits, region 2 is the largest (see MR2). In this scenario, companies with smaller margin difference are more likely to agree on a two-way contract (i.e., contract PAIE). In addition, when SI has sufficiently high marginal profit, region 1 is the smallest (see HR1). In this situation, SI has the most motivation to share emission reduction cost while SP is most unlikely to share advertising cost. 
Table 2 Supply chain members' optimal contract decisions regions under Model PAIE.

\begin{tabular}{|c|c|c|c|}
\hline Marginal profit & Relative CSR & SP's contract decision & SI's contract decision \\
\hline \multirow{3}{*}{$R_{S I} \leq \frac{R_{S P}}{2}$} & $0<\lambda \leq \frac{1}{3}$ & Y & $\mathrm{N}$ \\
\hline & $\frac{1}{3}<\lambda \leq \frac{2}{3}$ & $\mathrm{Y}$ & $\begin{array}{l}\theta \geq \bar{\theta}_{2}(\mathrm{Y}) \\
\theta<\bar{\theta}_{2}(\mathrm{~N})\end{array}$ \\
\hline & $\frac{2}{3}<\lambda \leq 1$ & $\begin{array}{l}\theta \leq \bar{\theta}_{1}(\mathrm{Y}) \\
\theta>\bar{\theta}_{1}(\mathrm{~N})\end{array}$ & $\begin{array}{l}\theta \geq \bar{\theta}_{2}(\mathrm{Y}) \\
\theta<\bar{\theta}_{2}(\mathrm{~N})\end{array}$ \\
\hline \multirow{3}{*}{$\frac{R_{S P}}{2}<R_{S I} \leq 2 R_{S P}$} & $0<\lambda \leq \frac{1}{3}$ & $\mathrm{Y}$ & $\begin{array}{l}\theta \leq \bar{\theta}_{2}(\mathrm{Y}) \\
\theta>\bar{\theta}_{2}(\mathrm{~N})\end{array}$ \\
\hline & $\frac{1}{3}<\lambda \leq \frac{2}{3}$ & $Y$ & $\mathrm{Y}$ \\
\hline & $\frac{2}{3}<\lambda \leq 1$ & $\begin{array}{l}\theta \leq \bar{\theta}_{1}(\mathrm{Y}) \\
\theta>\bar{\theta}_{1}(\mathrm{~N})\end{array}$ & $Y$ \\
\hline \multirow{3}{*}{$R_{S I}>2 R_{S P}$} & $0<\lambda \leq \frac{1}{3}$ & $\begin{array}{l}\theta \geq \bar{\theta}_{1} \\
\theta<\bar{\theta}_{1}\end{array}$ & $\begin{array}{l}\theta \leq \bar{\theta}_{2}(\mathrm{Y}) \\
\theta>\bar{\theta}_{2}(\mathrm{~N})\end{array}$ \\
\hline & $\frac{1}{3}<\lambda \leq \frac{2}{3}$ & $\begin{array}{l}\theta \geq \bar{\theta}_{1}(\mathrm{Y}) \\
\theta<\bar{\theta}_{1}(\mathrm{~N})\end{array}$ & Y \\
\hline & & $\mathrm{N}$ & Y \\
\hline
\end{tabular}

Notices: "Y" denotes "Yes" and "N" denotes "No", representing the players offering (not) positive cost-sharing rates.

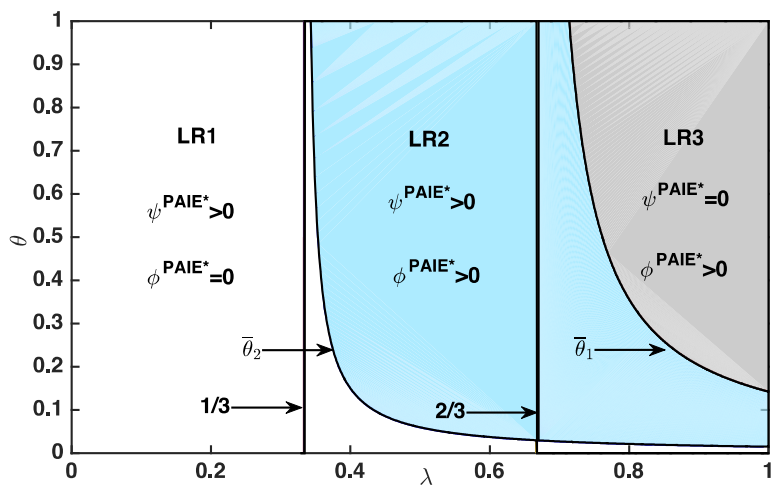

(a) $2 R_{S I} \leq R_{S P}$

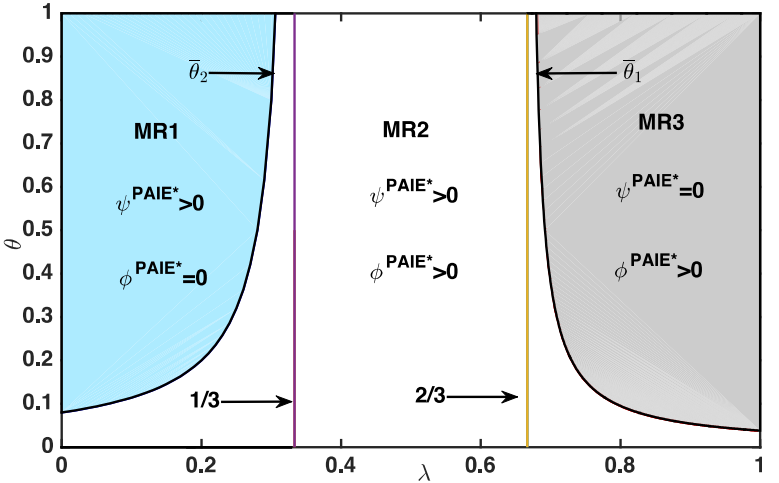

(b) $R_{S P} / 2<R_{S I} \leq 2 R_{S P}$

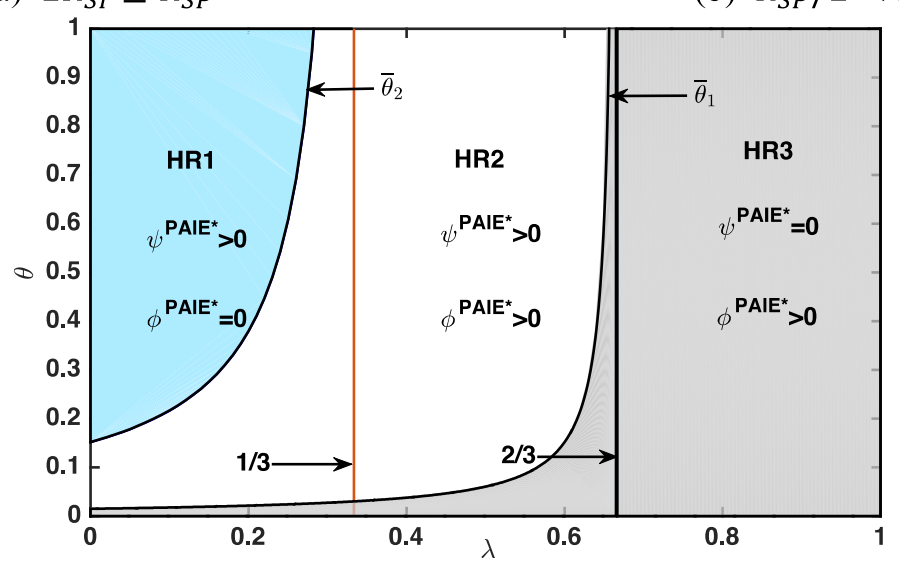

(c) $R_{S I}>2 R_{S P}$

Fig. 1. Supply chain members' optimal contract decisions regions under Model PAIE 
According to Lemma 2 and Proposition 3, we can derive the following Corollary 1 by comparing the cost-sharing rates of SP and SI when both of them implement cost-sharing contracts.

Corollary 1. When both SP and SI provide cost-sharing contract: if $0<\lambda \leq \min \left(\bar{\lambda}_{1}, 1\right), S P$ provides higher cost-sharing rate than that of SI (i.e., $\left.\varphi^{P A I E *} \geq \phi^{P A I E *}\right)$; if $\max \left(0, \bar{\lambda}_{1}\right)<\lambda \leq 1$, $S P$ provides lower cost-sharing rate than that of SI (i.e., $\varphi^{P A I E *}<\phi^{\text {PAIE*}}$ ), where $\bar{\lambda}_{1}=$ $\frac{\theta^{2} \mu_{a}+\gamma R_{S P}^{2}\left[\gamma \mu_{a}+\beta\left(\rho+\mu_{g}\right)\right]-\gamma R_{S I}^{2}\left[\gamma \mu_{a}+\beta\left(\rho+\mu_{g}\right)\right]+\theta R_{S P}\left[2 \gamma \mu_{a}+\beta\left(\rho+\mu_{g}\right)\right]}{\theta\left\{2 \theta \mu_{a}+\left(R_{S P}+R_{S I}\right)\left[2 \gamma \mu_{a}+\beta\left(\rho+\mu_{g}\right)\right]\right\}}$.

Corollary 1 reveals the effect of relative CSR on the optimal cost-sharing rates of SP and SI. It can be concluded that when SI has a lower CSR, SP would like to provide relatively high cost-sharing rate for SI. Conversely, SI prefers to provide higher cost-sharing rate. This is intuitive because the higher CSR the members have, the more utilities they can obtain from CSR so that they are more likely to participate in a low-carbon cooperation. Generally, chain member who has higher CSR is more likely to share higher cost of its partner, which is similar to the research result of Panda and Modak (2016).

\subsection{Equilibrium solutions under contract PAIS (Model PAIS)}

Under contract PAIS, both SP and SI first decide their cost-sharing rates, respectively. Afterwards, SP announces her service level and emission reduction effort, followed by SI who sets optimal advertising effort. The optimization control problems of them under contract PAIS are given as below.

$$
\begin{aligned}
\max _{S, E, \varphi} U_{S P}^{P A I S}= & \int_{0}^{\infty} e^{-\rho t}\left\{R_{S P} D[S(t), A(t), G(t)]+(1-\lambda) \theta G(t)-\frac{(1-\psi(t)) k_{S} S^{2}(t)}{2}\right. \\
& \left.-\frac{k_{e} E^{2}(t)}{2}-\frac{\phi(t) k_{a} A^{2}(t)}{2}\right\} d t
\end{aligned}
$$




$$
\begin{aligned}
\max _{A, \phi} U_{S I}^{P A I S}= & \int_{0}^{\infty} e^{-\rho t}\left\{R_{S I} D[S(t), A(t), G(t)]+\lambda \theta G(t)-\frac{(1-\psi(t)) k_{a} A^{2}(t)}{2}\right. \\
& \left.-\frac{\phi(t) k_{S} S^{2}(t)}{2}\right\} d t
\end{aligned}
$$

both are subject to Eq. (8).

Analogously, in Model PAIS, the optimal net utilities of SP and SI also satisfy the Hamilton-Jacobi-Bellman (HJB) equations as follows:

$$
\begin{aligned}
& \rho V_{S P}^{P A I S}=\max _{S, E, \varphi \geq 0}\left[R_{S P}(\alpha S+\beta A+G)+(1-\lambda) \theta G-\frac{(1-\phi) k_{S} S^{2}}{2}-\frac{k_{e} E^{2}}{2}-\frac{\psi k_{a} A^{2}}{2}\right. \\
& \left.\quad+V_{S P}^{P A I S \prime}\left(\mu_{a} A+\mu_{e} E-\mu_{g} G\right)\right] \\
& \rho V_{S I}^{P A I S}=\max _{A, \phi \geq 0}\left[R_{S I}(\alpha S+\beta A+\gamma G)+\lambda \theta G-\frac{(1-\psi) k_{a} A^{2}}{2}-\frac{\phi k_{S} S^{2}}{2}+V_{S I}^{\text {PAIS'}}\left(\mu_{a} A+\mu_{e} E\right.\right. \\
& \left.\left.-\mu_{g} G\right)\right]
\end{aligned}
$$

Solving the first-order conditions, we can obtain the chain members' optimal responsive decisions as follows.

$$
\left\{\begin{array}{c}
A=\frac{\beta R_{S I}+\mu_{a} V_{S I}^{P A I S \prime}}{(1-\varphi) k_{a}} \\
S=\frac{\alpha R_{S P}}{(1-\phi) k_{S}} \\
E=\frac{\mu_{e} V_{S P}^{P A I S \prime}}{k_{e}} \\
\varphi=\frac{\beta\left(2 R_{S P}-R_{S I}\right)+\mu_{a}\left(2 V_{S P}^{P A I S \prime}-V_{S I}^{P A I S \prime}\right)}{\beta\left(2 R_{S P}+R_{S I}\right)+\mu_{a}\left(2 V_{S P}^{P A I S \prime}+V_{S I}^{P A I S \prime}\right)} \\
\phi=\frac{\left(2 R_{S I}-R_{S P}\right)}{\left(2 R_{S I}+R_{S P}\right)}
\end{array}\right.
$$

Inserting Eq. (33) into Eqs. (31) and (32), the stationary feedback Stackelberg equilibrium solutions can be obtained. The following Lemma 3 summarizes these solutions.

Lemma 3. In Model PAIS, the feedback equilibrium solutions are expressed as follows. 


$$
\left\{\begin{array}{c}
S^{P A I S *}=\frac{\alpha R_{S P}}{\left(1-\phi^{P A I S *}\right) k_{S}} \\
E^{P A I S *}=\frac{\mu_{e}\left[(1-\lambda) \theta+\gamma R_{S P}\right]}{k_{e}\left(\rho+\mu_{g}\right)} \\
A^{P A I S *}=\frac{\beta\left(\rho+\mu_{g}\right) R_{S I}+\mu_{a}\left(\lambda \theta+\gamma R_{S P}\right)}{\left(1-\varphi^{P A I S *}\right)\left(\rho+\mu_{g}\right) k_{a}} \\
\varphi^{P A I S *}=\frac{\theta(2-3 \lambda) \mu_{a}+\left(2 R_{S P}-R_{S I}\right)\left[\gamma \mu_{a}+\beta\left(\rho+\mu_{g}\right)\right]}{\theta(2-\lambda) \mu_{a}+\left(2 R_{S P}+R_{S I}\right)\left[\gamma \mu_{a}+\beta\left(\rho+\mu_{g}\right)\right]} \\
\phi^{P A I S *}=\frac{\left(2 R_{S I}-R_{S P}\right)}{\left(2 R_{S I}+R_{S P}\right)}
\end{array}\right.
$$

The differential function of emission reduction can be deduced by substituting the above feedback equilibrium solutions into Eq. (1), resulting in $G(t)^{P A I S *}=G_{\infty}^{P A I S *}+\left(G_{0}-G_{\infty}^{P A I S *}\right) e^{-\mu_{g} t}$, wherein $G_{\infty}^{\text {PAIS* }}=\left(\mu_{e} / \mu_{g}\right) E^{\text {PAIS* }}+\left(\mu_{a} / \mu_{g}\right) A^{P A I S *}$. Substituting the above equilibrium solutions and state function into the objective functions Eqs. (29) and (30), we can obtain the optimal utilities of chain members shown as below:

$$
\begin{gathered}
U_{S P}^{P A I S *}=\frac{e^{-\rho t}\left\{\left\{2 k_{a}\left[2 k_{s} M_{1}^{2} \mu_{e}^{2}+\alpha^{2} k_{e} R_{m}\left(R_{m}+2 R_{r}\right)\left(\rho+\mu_{g}\right)^{2}\right]+k_{e} k_{S} M_{2}^{2}\right\} / k_{S}+\left[4 M_{1}\left(2 k_{a} M_{1} \mu_{e}^{2}+k_{e} \mu_{a} M_{2}\right)\right] / \gamma\right\}}{8 k_{a} k_{e}\left(\rho+\mu_{g}\right)^{2}} \\
U_{S I}^{P A I S *}=\frac{e^{-\rho t}\left\{k_{a}\left[8(1+\gamma) k_{S} M_{1}\left(\theta \lambda+\gamma R_{r}\right) \mu_{e}^{2}+\alpha^{2} \gamma k_{e}\left(R_{m}+2 R_{r}\right)^{2}\left(\rho+\mu_{g}\right)^{2}\right]+2 k_{e} k_{S} M_{2} M_{4}\right\}}{8 \gamma k_{a} k_{e} k_{S}\left(\rho+\mu_{g}\right)^{2}}
\end{gathered}
$$

From Lemma 3, it can be found that consumers' low-carbon preference, CSR and relative CSR cannot influence SI's service cost-sharing rate. However, the marginal profits of SP and SI can make significant impacts. Specifically, SI will increase his service cost sharing fraction as his own margin arises but decreases with the increase of SP's margin. As a result, when SI adopts contract PAIS, he just needs to care about the marginal profits of herself and SP to set a participation rate.

Proposition 4. In Model PAIS, the cost sharing rates decisions of SP and SI can be summarized as follows, where $\bar{\theta}_{1}=\frac{\left(2 R_{S P}-R_{S I}\right)\left[\gamma \mu_{a}+\beta\left(\rho+\mu_{g}\right)\right]}{(3 \lambda-2) \mu_{a}}$.

(i) When $R_{S I} \leq R_{S P} / 2$, SI always does not share service cost. For $S P$, if $0 \leq \lambda \leq 2 / 3$, she always shares advertising cost; if $2 / 3<\lambda \leq 1$ and $\theta \leq \bar{\theta}_{1}$, she would share advertising cost, otherwise no sharing.

(ii) When $R_{S P} / 2<R_{S I} \leq 2 R_{S P}$, SI always shares service cost. For $S P$, if $0 \leq \lambda \leq 2 / 3$, she 
always shares advertising cost; if $2 / 3<\lambda \leq 1$ and $\theta \leq \bar{\theta}_{1}$, SP would share advertising cost, otherwise no sharing.

(iii) When $R_{S I}>2 R_{S P}$, SI always shares service cost. For $S P$, if $0 \leq \lambda \leq 2 / 3$ and $\theta \geq \bar{\theta}_{1}$, she would share advertising cost, otherwise no sharing; if $2 / 3<\lambda \leq 1$, SP would not share any advertising cost.

Fig. 2 is drawn to contribute to our explanations. From Proposition 4, we can understand that marginal profits, CSR and relative CSR play important roles in designing contracts. From the marginal profits' perspective, when SI has a sufficiently low marginal profit, he never shares SP's service cost. In this case, SP would provide a cost-sharing contract in most of the regions expect for the case of higher CSR and relative CSR. When there is no big gap between SP's and SI's marginal profits, SI always shares the service cost and SP's contract decision is similar to that in the former situation. When SI has a sufficiently high margin, he also always provides a cost-sharing contract. SP offers a positive participation rate only when the CSR of the SC system is relatively high and relative CSR is low enough. In this case, SP will have the lowest motivation to implement a cost-sharing contract. In conclusion, SI would support SP's emission reduction effort only if a condition on margins is met, namely, SI's marginal profit is at least half of SP's.

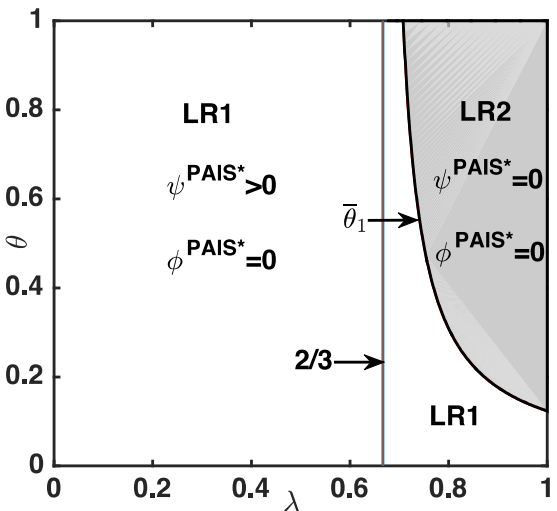

(a) $2 R_{S I} \leq R_{S P}$

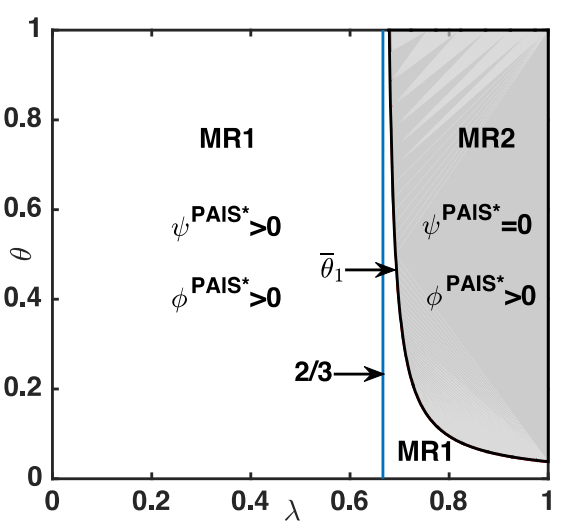

(b) $R_{S P} / 2<R_{S I} \leq 2 R_{S P}$

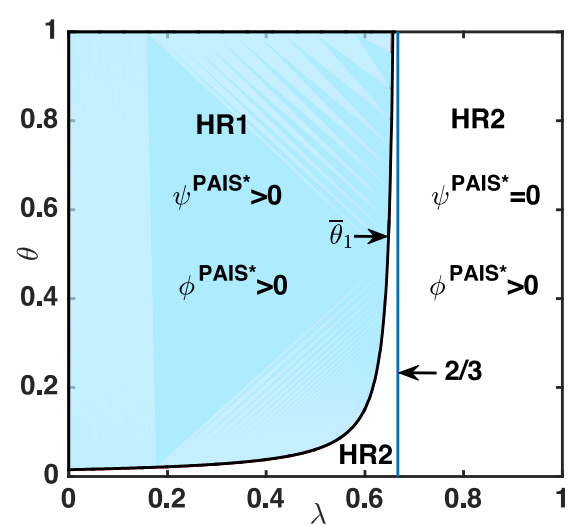

(c) $R_{S I}>2 R_{S P}$

Fig. 2. Supply chain members' optimal contract decisions regions under Model PAIS

Comparing the cost-sharing rates of SP and SI under contract PAIS, we can derive the following Corollary 2. We refer the readers to Corollary 1 for getting more similar properties. 
Corollary 2. When both SP and SI provide cost-sharing contract, if $0<\lambda \leq \min \left(\bar{\lambda}_{2}, 1\right)$, SP provides higher cost-sharing rate than that of SI (i.e., $\left.\varphi^{P A I S *} \geq \phi^{P A I S *}\right)$; if $\max \left(0, \bar{\lambda}_{2}\right)<\lambda \leq 1$, $S P$ undertakes lower cost-sharing rate than that of SI (i.e., $\left.\varphi^{P A I S *}<\phi^{\text {PAIS* }}\right)$, where $\bar{\lambda}_{2}=$ $\frac{\theta \mu_{a} R_{S P}+\left(R_{S P}^{2}-R_{S I}^{2}\right)\left[\gamma \mu_{a}+\beta\left(\rho+\mu_{g}\right)\right]}{\theta\left(R_{S P}+R_{S I}\right) \mu_{a}}$.

\section{Comparison among different cost-sharing contracts}

This section mainly compares the feedback equilibrium solutions. The purpose is to see how different cost-sharing contracts affect the optimal outcomes. In the analysis that follows, we start by comparing the optimal service levels, emission reduction and advertising efforts among three models, resulting in Proposition 5.

Proposition 5. The optimal service levels, emission reduction and advertising efforts have the following relationships:

(i) When $2 R_{S I} \geq R_{S P}$, we have $S^{P A I S *} \geq S^{P A *}=S^{P A I E *}$. While $2 R_{S I}<R_{S P}$, we have $\phi^{\text {PAIS* }}=$ 0 so that $S^{P A *}=S^{P A I E *}=S^{P A I S *}$.

(ii) When $0 \leq \lambda \leq \frac{1}{3} \& \theta \leq \min \left(\bar{\theta}_{2}, 1\right)$ or $\frac{1}{3}<\lambda \leq 1 \& \theta>\max \left(0, \bar{\theta}_{2}\right), \quad E^{P A I E *} \geq E^{P A *}=$ $E^{P A I S *}$, otherwise $\phi^{P A I E *}=0$ and $E^{P A *}=E^{P A I S *}=E^{P A I E *}$.

(iii) Under three models, $A^{P A *}=A^{P A I E *}=A^{P A I S *}$.

According to Proposition 5, it can be concluded that different cooperative ways do not change SP's advertising cost-sharing rates. By contrast, various cooperative contracts could influence the optimal service level and emission reduction effort under certain conditions. To be specific, Proposition 5(i) highlights that when SI has a sufficiently high marginal profit, his service cost-sharing contract can encourage SP to provide a higher service level. In addition, when the joint effect of CSR and relative CSR is relatively low or high, SI sharing SP's emission reduction cost can stimulate SP to offer a higher emission reduction effort than that under Model PA, otherwise SI 
would not provide an emission reduction cost-sharing contract, thereby leading to the same emission reduction effort. In short, we conclude that the two-way contracts indeed improve the corresponding service level and emission reduction effort as long as SI is willing to provide positive participation rates.

Corollary 3. When $0 \leq \lambda \leq \frac{1}{3} \& \theta \leq \min \left(\bar{\theta}_{2}, 1\right)$ or $\frac{1}{3}<\lambda \leq 1 \& \theta>\max \left(0, \bar{\theta}_{2}\right), G^{\text {PAIE* }} \geq$ $G^{P A *}=G^{P A I S *}$, otherwise $\phi^{P A I E *}=0$ and $G^{P A *}=G^{P A I S *}=G^{P A I *}$.

From Corollary 3, it is obvious that emission reduction levels under Model PA and Model PAIS are always equal. Similar to emission reduction effort, if both relative CSR of SI and CSR of the service SC are sufficiently low or high, SI sharing SP's emission reduction cost is better off for improving environment. When both of them are moderate, there is no motivation for SI to share the emission reduction cost. In a special case that the marginal profit of SI is equal to the half of SP's, only when the relative CSR is high enough $(\lambda \geq 1 / 3)$ can the contract PAIE benefit the environment.

In the following part, we will explore how supply chain members decide their own participation rates.

Proposition 6. The optimum cost-sharing rates of SP and SI adhere to the following relationships:

(i) $\varphi^{P A *}=\varphi^{P A I E *}=\varphi^{P A I S *}$.

(ii) When $R_{S P} / R_{S I} \geq(1-\lambda) / \lambda$, $\phi^{\text {PAIE* }} \geq \phi^{\text {PAIS* }}$, otherwise $\phi^{\text {PAIE* }}<\phi^{\text {PAIS* }}$.

From Proposition 6(i), SP always adopts the identical advertising cost-sharing rate. The intuitive reason is that we focus on investigating different cost-sharing contracts of SI. Under such circumstance, SP can adjust her service level or emission reduction effort as respond to SI's contract decisions instead of adjusting participation rate. It is clearly seen from Proposition 6(ii) that marginal profit ratio and relative CSR ratio between SP and SI have great impacts on SI's cost-sharing contract decisions. In particular, if marginal profit ratio is not less than relative CSR 
ratio, SI will bear higher cost sharing rate in contract PAIE than that in contract PAIS. Conversely, he will offer a lower rate when relative CSR ratio is over than their marginal profit ratio. The reason behind is that higher marginal profit ratio implies that SI has a relative advantage of CSR but has a disadvantage of marginal profit compared to SP. Therefore, SI has more incentive to offer higher emission reduction cost-sharing rate so as to stimulate SP to conduct emission reduction activity. Otherwise, directly offering higher service sharing cost rate is better for SI.

Next, we turn our attention to compare the supply chain's and its members' utilities. From Proposition 4(i), we know that SI always does not share the service cost when $R_{S P}>2 R_{S I}$. We first focus on identifying the conditions under what and which contract is the best choice when the three contracts can coexist under the precondition $R_{S P} \leq 2 R_{S I}$. To facilitate exposition, we define $\Omega \equiv\{(\lambda, \theta) \mid 0 \leq \lambda \leq 1,0 \leq \theta \leq 1\}$ as the universal set.

Proposition 7. Given $R_{S P} \leq 2 R_{S I}$ and $(\lambda, \theta) \in \Omega$, the optimal utilities of SP satisfy the following relationships:

(i) When $(\lambda, \theta) \in R_{1}$, if $k_{S} \leq \bar{k}_{S 1}$, then $U_{S P}^{P A I S *} \geq U_{S P}^{P A I E *} \geq U_{S P}^{P A *}$, if $k_{S}>\bar{k}_{S 1}$, then $U_{S P}^{P A I E *}>$ $U_{S P}^{P A I S *} \geq U_{S P}^{P A *}$. When $(\lambda, \theta) \in R_{2}$, then $\phi^{P A I E *}=0$ and $U_{S P}^{P A I S *}>U_{S P}^{P A *}=U_{S P}^{P A I E *}$.

(ii) When $(\lambda, \theta) \in R_{3}$, if $k_{S} \leq \bar{k}_{S 1}$, then $U_{S P}^{P A I S *} \geq U_{S P}^{P A I E *} \geq U_{S P}^{P A *}$, if $k_{S}>\bar{k}_{S 1}$, then $U_{S P}^{P A I E *}>$ $U_{S P}^{P A I S *} \geq U_{S P}^{P A *}$

where $\bar{k}_{s 1}=\frac{\alpha^{2} k_{e} R_{S P}\left(2 R_{S I}-R_{S P}\right)\left(\rho+\mu_{g}\right)^{2}}{\left[\theta(1-\lambda)+\gamma R_{S P}\right]\left[\gamma\left(2 R_{S I}-R_{S P}\right)-\theta(1-3 \lambda)\right] \mu_{e}^{2}}, R_{1} \equiv\left\{(\lambda, \theta) \mid 0 \leq \lambda \leq \frac{1}{3}, 0<\theta \leq \min \left(1, \bar{\theta}_{2}\right)\right\}$, $R_{2} \equiv\left\{(\lambda, \theta) \mid 0 \leq \lambda \leq \frac{1}{3}, \max \left(0, \bar{\theta}_{2}\right)<\theta<1\right\}$, and $R_{3} \equiv\left\{(\lambda, \theta) \mid \frac{1}{3}<\lambda \leq 1,0 \leq \theta \leq 1\right\}$

Proposition 7 identifies the conditions under which SP can obtain more utility. It would say that when SI has a relatively high marginal profit, contracts PAIS and PAIE would be probably the best choice for SP. And CSR, relative CSR, and SP's service cost efficiency significantly affect SP's optimal utility. To be more specific, when the joint effect of CSR and relative CSR is adequately low, SI can benefit SP by offering any one of the two-way contracts. In this setting, if SP has higher 
service efficiency, contract PAIS is better for SP; while contract PAIE is more beneficial to SP under a lower service efficiency. Notice that if the CSR is sufficiently high, SI will have no motivation to adopt a cost-sharing contract based on SP's emission reduction cost. From Proposition 7(ii), SI always wants to provide two-way contracts regardless of the value of parameter $\theta$ when he has a high enough CSR preference.

Proposition 8. The SI's optimal utilities across the three models hold the following ordering relationships, where $\bar{k}_{S 2}=\frac{\alpha^{2} k_{e}\left(R_{S P}-2 R_{S I}\right)^{2}\left(\rho+\mu_{g}\right)^{2}}{\left[\theta(1-3 \lambda)+\gamma\left(R_{S P}-2 R_{S I}\right)\right]^{2} \mu_{e}^{2}}$.

(i) When $0<k_{s} \leq \bar{k}_{S 2}$, we have $U_{S I}^{P A I S *} \geq U_{S I}^{P A I E *} \geq U_{S I}^{P A *}$.

(ii) When $k_{S}>\bar{k}_{S 2}$, we have $U_{S I}^{P A I E *}>U_{S I}^{P A I S *} \geq U_{S I}^{P A *}$.

Proposition 8 highlights that SI providing cost-sharing contract (if $\phi^{*}>0$ ) is always better for herself no matter which cost-sharing contract he selects. More specifically, when SP's service cost efficiency is sufficiently high (i.e., $k_{s}$ is lower), he would provide SP with a cost subsidy based on the service cost-sharing contract. However, if SP's service cost efficiency is lower (i.e., higher $k_{s}$ ), contract PAIE is better off for SI. To sum up, SI makes a contract decision depending largely on SP's service cost sensitivity coefficient.

According to Propositions 7 and 8, we can derive the following results that reveal the equilibrium contract decisions of SP and SI.

Corollary 4. Given $R_{S P} \leq 2 R_{S I}$ and $(\lambda, \theta) \in \Omega$, SP and SI can achieve an equilibrium contract under the following conditions:

(i) When $(\lambda, \theta) \in R_{1}$, if $k_{s} \leq \bar{k}_{s 1}$, contract PAIS is the equilibrium contract; if $k_{s}>\bar{k}_{s 2}$, contract PAIE is the equilibrium contract.

(ii) When $(\lambda, \theta) \in R_{2}$ and $k_{s} \leq \bar{k}_{s 2}$, contract PAIS is the equilibrium contract.

(iii) When $(\lambda, \theta) \in R_{3}$ and $R_{S P} / R_{S I} \leq(1-\lambda) / \lambda$, if $k_{S} \leq \bar{k}_{s 1}$, contract PAIS is the equilibrium contract; if $k_{S}>\bar{k}_{s 2}$, contract PAIE is the equilibrium contract. 
(iv) When $(\lambda, \theta) \in R_{3}$ and $(1-\lambda) / \lambda<R_{s p} / R_{S I}$, if $k_{s}<\bar{k}_{s 2}$, contract PAIS is the equilibrium contract; if $k_{S}>\bar{k}_{s 1}$, contract PAIE is the equilibrium contract.

Corollary 4 points out the same contract preferences of SP and SI under precondition $R_{S P} \leq 2 R_{S I}$. In other words, both SP and SI can benefit more from the equilibrium contract, which can ensure the long-term stability of their cooperation. In general, when SP has a relatively high cost efficiency of service (i.e., $k_{S}$ is lower), contract PAIS would be the equilibrium contract. However, contract PAIE would be the equilibrium contract when SP has a lower service cost efficiency (i.e., $k_{s}$ is higher). Note that when parameter $\lambda$ is lower but $\theta$ is higher, SI would have no motivation to provide contract PAIE so that only contract PAIS would be the potential equilibrium contract.

Proposition 9. Given $R_{S P} \leq 2 R_{S I}$ and $(\lambda, \theta) \in \Omega$, the relationships of optimal utilities of the whole supply chain hold as follows:

(i) When $(\lambda, \theta) \in R_{1}$, if $k_{s} \leq \bar{k}_{S 3}$, then $U_{S C}^{P A I S *} \geq U_{S C}^{P A I E *} \geq U_{S C}^{P A *}$; if $k_{s}>\bar{k}_{s 3}$, we have $U_{S C}^{P A I E *}>U_{S C}^{P A I S *} \geq U_{S C}^{P A *}$. When $(\lambda, \theta) \in R_{2}$, then $U_{S C}^{P A I S *} \geq U_{S C}^{P A *}=U_{S C}^{P A I E *}$.

(ii) When $(\lambda, \theta) \in R_{3}$, if $k_{S} \leq \bar{k}_{S 3}, U_{S C}^{P A I S *} \geq U_{S C}^{P A I E *}>U_{S C}^{P A *}$; if $k_{S}>\bar{k}_{S 3}$, we have $U_{S C}^{P A I E *}>$ $U_{S C}^{P A I S *} \geq U_{S C}^{P A *}$

where $\bar{k}_{S 3}=\frac{\alpha^{2} k_{e}\left(R_{S P}^{2}-4 R_{S I}^{2}\right)\left(\rho+\mu_{g}\right)^{2}}{\left[\theta^{2}(1+\lambda)(1-3 \lambda)+2 \gamma \theta(1-\lambda) R_{S P}+\gamma^{2} R_{S P}^{2}-8 \gamma \theta \lambda R_{S I}-4 \gamma^{2} R_{r}^{2}\right] \mu_{e}^{2}}$.

According to Proposition 9, we identify the conditions under which the entire SC can obtain the highest utility. As per Proposition 9(i), when SI can earn higher marginal profit and both the CSR and relative CSR are relatively low, the service SC can get more utilities when SI provides either of the two-way cost-sharing contracts. In this case, higher SP's service cost efficiency often brings more utility under contract PAIS than contract PAIE. In a smaller situation $(\lambda, \theta) \in R_{2}$, SI is unwilling to provide contract PAIE so that only contract PAIS may be beneficial to the whole SC. Similar to Proposition 7(ii), when SI has relatively high enough CSR (i.e., $\lambda \geq 1 / 3$ ), selecting 
two-ways contracts always benefit the service SC no matter what the parameter $\theta$ is.

Now, we take the case $R_{S P}>2 R_{S I}$ into consideration. In this situation, we just need to compare contracts PA and PAIE.

Proposition 10. Given $R_{S P}>2 R_{S I}$ and $(\lambda, \theta) \in \Omega$, the relationships of optimal utilities of SP, SI and the whole supply chain hold as follow: when $(\lambda, \theta) \in R_{1}$, then $U_{i}^{P A I E *} \geq U_{i}^{P A *} ;$ when $(\lambda, \theta) \in R_{2}$, then $\phi^{P A I E *}=0$ so that $U_{i}^{P A I E *}=U_{i}^{P A *} ;$ when $(\lambda, \theta) \in R_{3}$, we have $U_{i}^{P A I E *}>U_{i}^{P A *}$, where $i=S P, S I, S C$.

Proposition 10 demonstrates that when SI has a sufficiently low margin, he has no motivation to adopt contract PAIS. In this setting, SI using contract PAIE would always benefit the whole service SC and its members. Put differently, given that $R_{S P}>2 R_{S I}$, contract PAIE is a dominant contract at the most of situations except for a special case where the parameter $\lambda$ is lower while $\theta$ is relatively high so that SI has no incentive to adopt contract PAIE. In the small situation, only contract PA may be the feasible contract for the SC. Consequently, it can be suggested that chain members should adopt contract PAIE to achieve Pareto improvement when SI has a relatively low marginal profit as long as he is willing to provide this contract. Otherwise, contract PA is the only potential choice.

\section{Numerical and sensitivity analysis}

In this section, we perform numerical simulations and sensitivity analyses to better understand the effects of some other critical system parameters on the theoretical results. The following parameter values are selected based on several earlier studies (Liu, Anderson, \& Cruz, 2012; Zhang et al., 2017; $\mathrm{Zu}$ et al., 2018), and we further normalize them as benchmarks: $R_{S P}=0.8, \lambda=$ $0.5, \alpha=0.5, \quad \beta=0.3, \gamma=0.5, \quad \rho=0.1, \mu_{e}=0.6, \mu_{a}=0.7, \quad \mu_{g}=0.1, k_{a}=0.4, \quad k_{e}=0.3$, $k_{s}=0.4, R_{S I}=0.6$ and $\theta=0.5$. We aim at analyzing the effects of a specific factor under given other parameters based on the benchmark setting. 


\subsection{Effect analysis of factors $\mu_{a}, \mu_{e}, \mu_{g}$}

In this subsection, we aim to scrutinize the influences of parameters $\mu_{a}, \mu_{e}$ and $\mu_{g}$ on the utilities of supply chain members and the performance of the entire service SC.
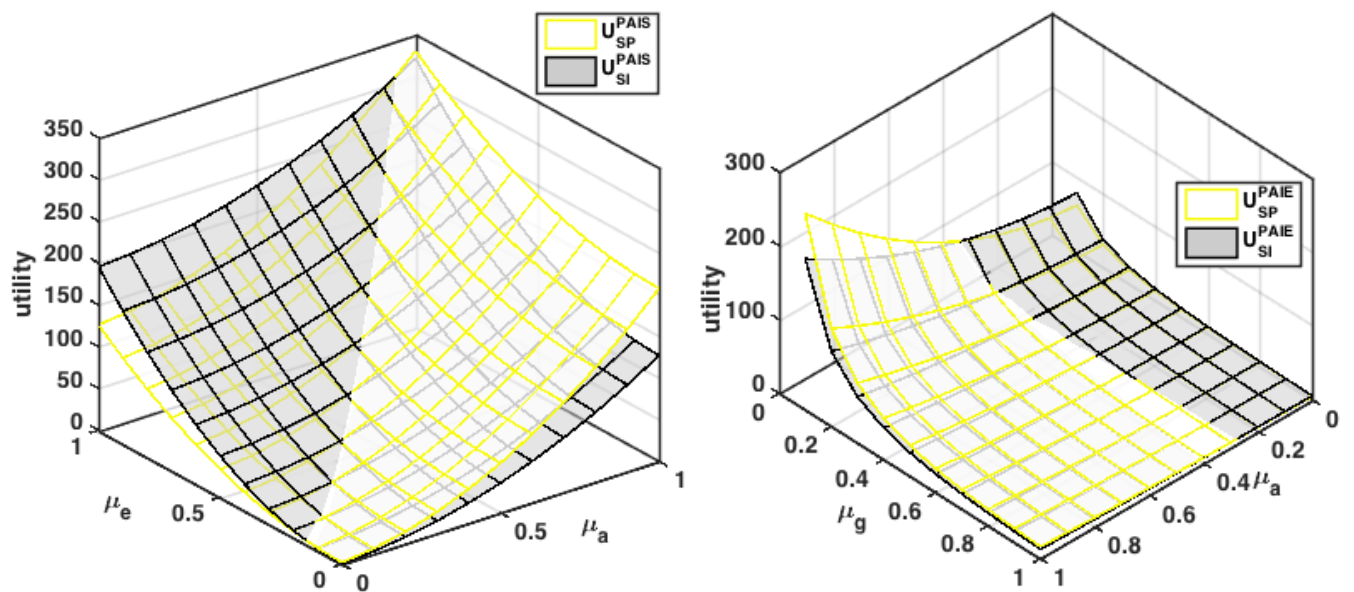

Fig. 3. The effects of factors $\mu_{a}, \mu_{e}$ and $\mu_{g}$ on SP's and SI's utilities
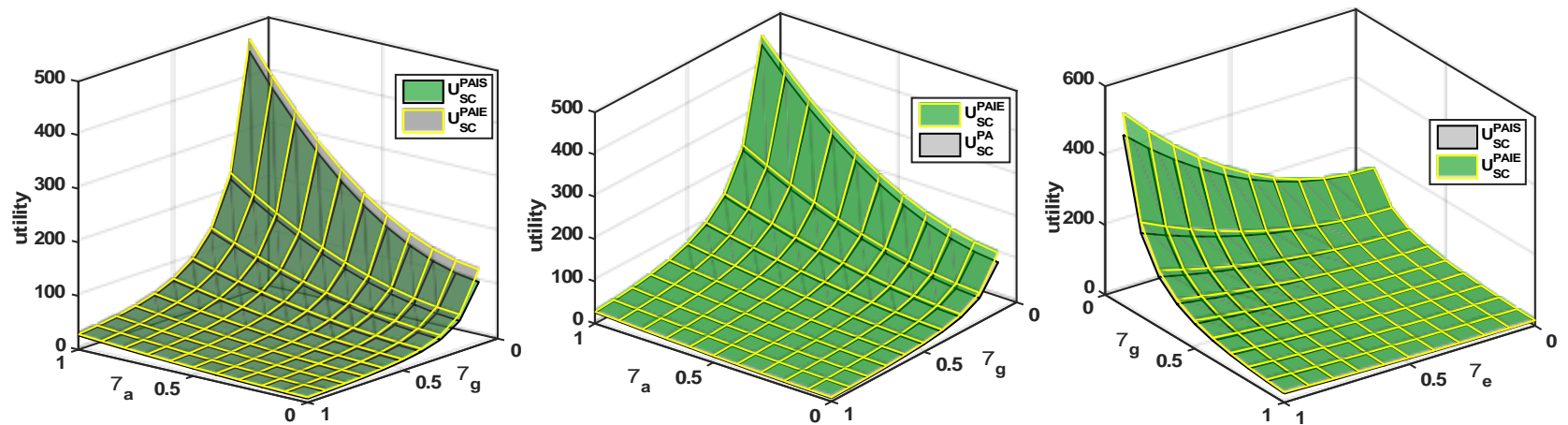

Fig. 4. The effects of factors $\mu_{a}, \mu_{e}$ and $\mu_{g}$ on supply chain performance

As shown in Figs. 3 and 4, it can be clearly found that the factors related to sate function of emission reduction strongly affect players' utilities and the entire service supply chain's utility. In particular, the sensitivity coefficients $\mu_{a}$ and $\mu_{e}$ play positive roles in promoting the utilities of chain members and the whole service SC, while the parameter $\mu_{g}$ plays a negative role. In other words, when SI's advertising and SP's emission reduction efforts are more beneficial to improve the emission reduction, service firms are more likely to take part in carbon emission reduction activities. By contrast, a higher decay rate of emission reduction will restrain the implementation of carbon emission reduction. Therefore, the government should strengthen publicity propaganda of low-carbon services to improve the consumers' environmental awareness. 


\subsection{Effect analysis of factors $\alpha, \beta, \gamma$}

Similarly, using the basic parameters setting, we next focus on investigating the impacts of $\alpha$, $\beta$ and $\gamma$ on emission reduction and utilities of the service SC members. We here just present some representative illustrations and omit some very similar results to save space.
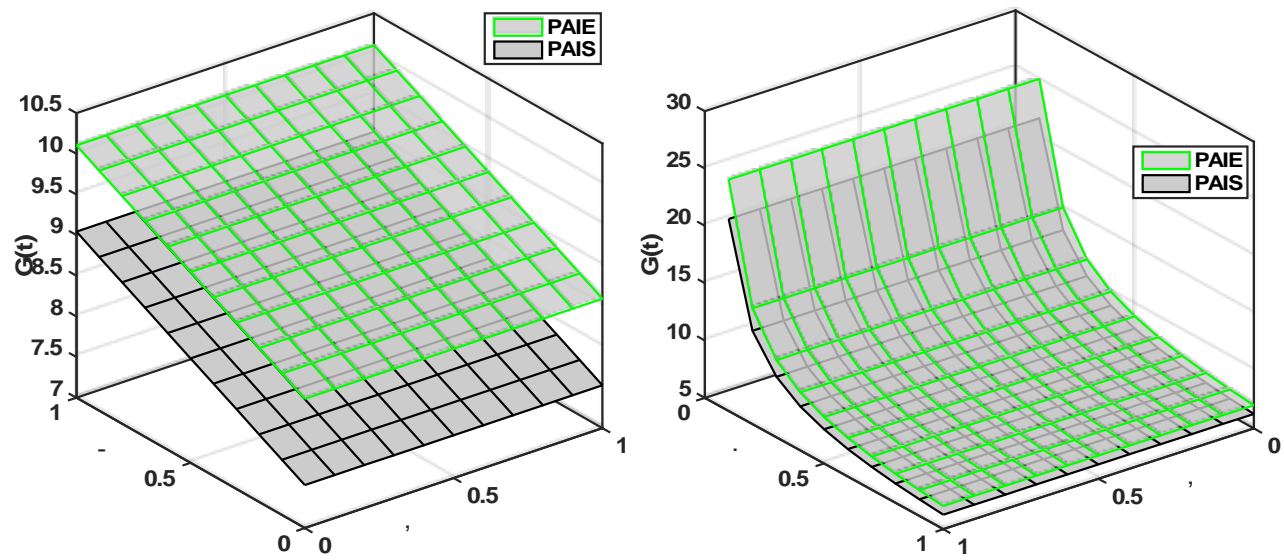

Fig. 5. The effects of factors $\alpha, \beta$ and $\gamma$ on emission reduction amount
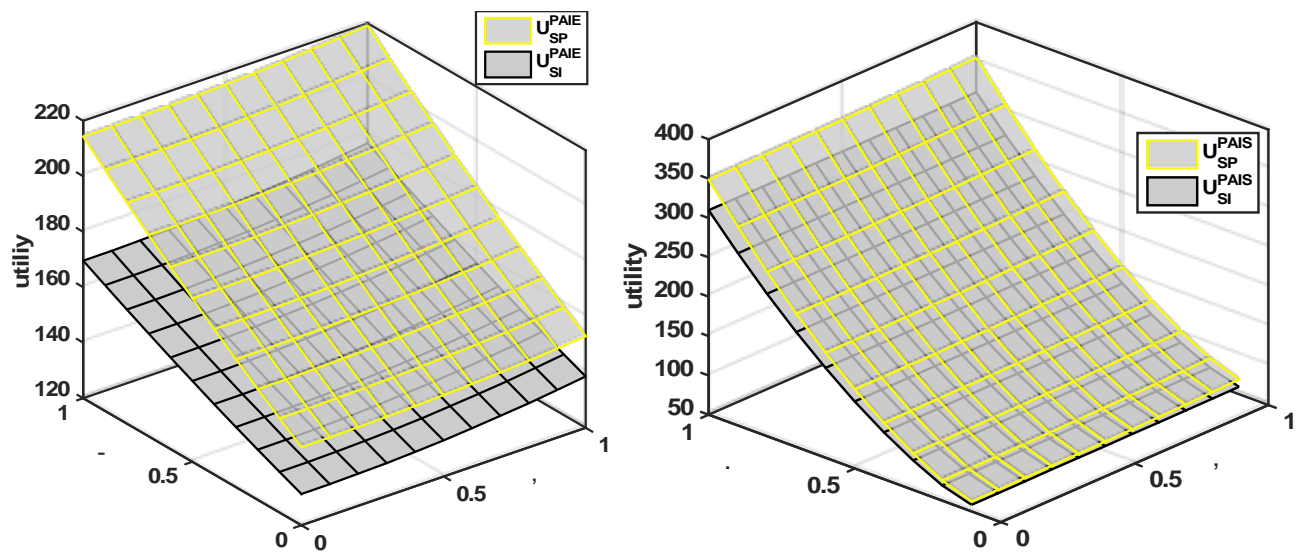

Fig. 6. The effects of factors $\alpha, \beta$ and $\gamma$ on utilities of supply chain members

It is known from Figs. 5 and 6 that higher service sensitivity coefficient has almost no impact on emission reduction, but it indeed can improve the chain members' utilities. In other words, when consumers become increasingly sensitive to the service, it can just contribute to enhancing the firms' utilities but cannot promote the low-carbon activity. Additionally, it can be seen that the carbon emission reduction and advertising coefficients have significant effects on the emission reduction and SC participants' utilities. In particular, the emission reduction is sharply increasing as parameters $\beta$ and $\gamma$ increase. This means when the consumers are more sensitive to the advertising and firms' emission reduction efforts, it is more likely to promote the development of 
low-carbon service industry. Similarly, when factors $\beta$ and $\gamma$ increase, the chain members can obtain more utility, thereby resulting in higher SC utility.

\subsection{Effect analysis of cost coefficients $k_{s}, k_{a}, k_{e}$}

In this subsection, we investigate the effects of cost sensitivity parameters $k_{s}, k_{a}, k_{e}$ based on the previous parameters values. The illustration results are presented in the following figures.
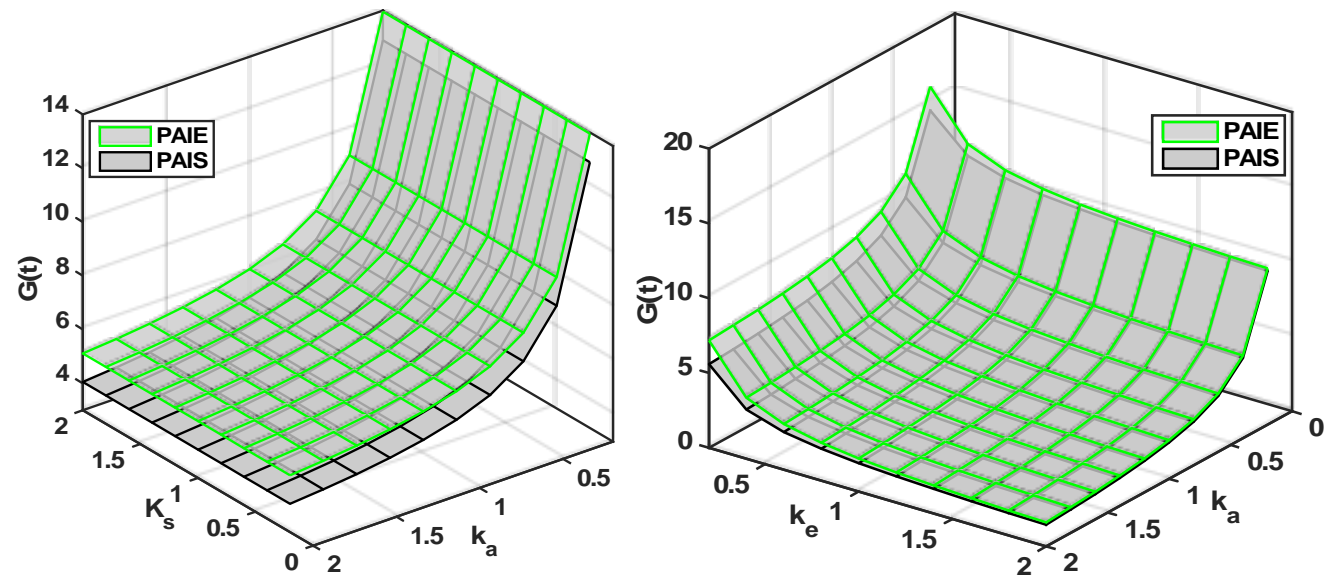

Fig. 7. The effects of coefficients $k_{s}, k_{a}$ and $k_{e}$ on emission reduction amount.
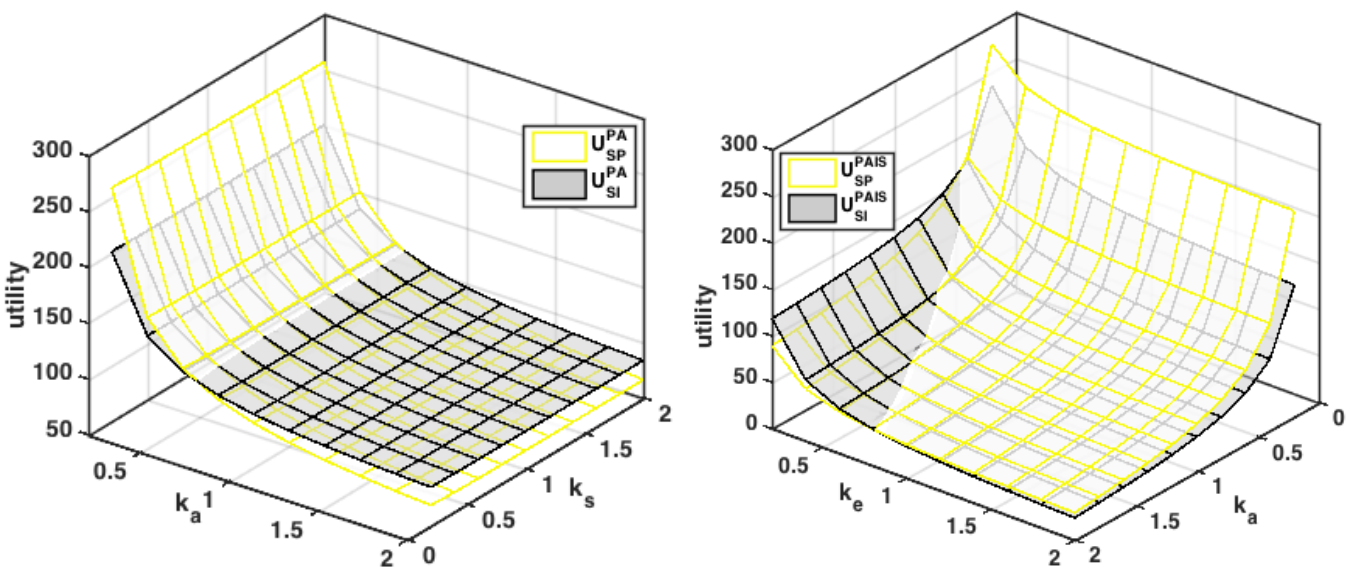

Fig. 8. The effects of coefficients $k_{\mathrm{s}}, k_{a}$ and $k_{e}$ on utilities of supply chain members.

According to Figs. 7 and 8, it can be obviously observed that SP's service cost efficiency and consumers' low-carbon preference, as well as SI's advertising cost efficiency can largely influence the carbon emission reduction amount and the chain members' utilities. The effect of each type of cost efficiency parameters is very similar. On the whole, when the cost efficiency is getting worse (i.e., higher $k_{a}$ or $k_{e}$ ), both the emission reduction and utility of each chain member are getting lower. As to SP's service cost efficiency $k_{s}$, we can clearly see that it does not affect the emission 
reduction. However, it does work on the utilities of the whole service SC and its members. These results are relatively intuitive because less cost investment is needed to get the same emission reduction under higher service efficiency and there is no service-related factor into the state function of emission reduction.

\section{Conclusions and managerial implications}

In the context of low-carbon economic development, we consider a service supply chain comprised of a service provider who is responsible to conduct carbon emission reduction and a service integrator who is in charge of promoting the low-carbon activities in the presence of CSR. We focus on discussing SI's three possible cost sharing decisions, namely, not sharing any cost of SP, sharing SP's emission abatement cost and sharing SP's service cost. According to Hamilton-Jacobi-Bellman equations, we derive the optimal outcomes based on utility maximization principle. Furthermore, we compare the feedback equilibrium outcomes across the three different contracts. This research makes the following major contributions.

Firstly, this study incorporates consumers' low-carbon preference into the low-carbon service SC from a differential game's perspective. Our paper verifies that optimal emission reduction and low-carbon advertising efforts are always positively proportional to the consumers' low-carbon preference. Hence, service enterprises should strengthen the propagation of information regarding low-carbon/eco-friendly activities in order to improve consumers' environmental protection consciousness. Differently from the previous studies, our paper further shows that the optimal cost-sharing rates of supply chain members and consumers' low-carbon preference have not a simple monotone correlation relationship but a relative complex relationship. This suggests that service firms should design appropriate cost-sharing rates for their partners on the basis of different consumers' low-carbon preference.

Secondly, taking account of the service firms' CSR behaviors generates an alternative avenue for profit maximization in service supply chains management. Deviating from the 
profit-maximizing principle, our paper confirms that enterprises' CSR behaviors have significant influences on optimal service level, emission reduction and advertising efforts. The outcome of this research shows that higher CSR invariably motivates chain members to provide higher emission reduction and advertising efforts under three contracts. This means that the whole supply chain's CSR indeed contributes to the development of low-carbon economy. Additionally, SI adopting contract PAIE can change the effect trend of SI's relative CSR compared to contracts PA and PAIS where higher relative CSR would lead to lower emission reduction and advertising efforts. While in contract PAIE, they are increasing in relative CSR. This implies that contract PAIE can eliminate the environmental preference conflict between upstream and downstream firms in a service SC system, which is conducive to supply chain members for agreeing on an identical contract.

Lastly, the study results provide some guidelines on setting service, emission reduction and advertising for service firms and contribute a theoretical basis for the cost-sharing contract design for a low-carbon service SC when chain members have CSR behaviors. It concludes that two-way cost-sharing contracts could be beneficial to the whole SC and its members but it does not mean that the decision-maker is always willing to adopt a two-way contract. Sometimes contract PA may be the unique feasible contract. When SI is willing to take the two-way contracts, we find that the service cost sensitivity coefficient is the main factor that would affect the contract decision. Furthermore, when SP's service efficiency is lower, SI prefers to adopt contract PAIE. Otherwise, contract PAIS is better for him. More importantly, contracts PAIS and PAIE would be the potential equilibrium contract when SI's marginal profit is relatively high. However, when it is lower contracts PAIE and PA would be the possible equilibrium contract. Therefore, it can be suggested that service SC members should choose an appropriate contract to maximize their expected utilities depending on their marginal profits, CSR, relative CSR and service cost efficiency as well as consumers' low-carbon preference.

Despite our research's several major contributions to the literature on low-carbon economic and cost-sharing contract design, there are still some limitations and deficiencies for future research 
to address. Firstly, our paper focuses on discussing three different cost-sharing contracts. Hence, future research can be done on other frequently used coordinative contracts (e.g., revenue/profit sharing contract, quantity discount contract and quantity flexibility contract). Secondly, our results are established by assuming completely symmetric information. In practice, cost and/or demand information may be private information. Therefore, considering the possibility of asymmetric information could be another possible future research direction. Finally, our paper assumes deterministic demand. So, further research can consider stochastic market demand in modeling and analysis.

\section{Appendix A. Proofs of Lemmas}

\section{Proof of Lemma 1}

According to Eq. (10), it can be deduced that $\frac{\partial^{2} U_{S I}^{P A}}{\partial A^{2}}=-(1-\varphi) k_{a}<0$, which means the utility function of SI is concave in $A$. In addition, the Hessian matrix of the SP's utility function is $H(S, E)_{S P}^{P A}=\left[\begin{array}{cc}-k_{S} & 0 \\ 0 & -k_{e}\end{array}\right]$. Therefore, we can have $\left|H(S, E)_{S P}^{P A}\right|>0$ and the first-order sequence principal minor is negative. Hence, we can obtain Eq. (11) by solving the following first-order conditions.

$k_{S} S-\alpha R_{S P}=0 ;(1-\varphi) k_{a} A-\beta R_{S I}+\mu_{a} V_{S I}^{P A^{\prime}}=0 ; k_{e} E-\mu_{e} V_{S P}^{P A^{\prime}}=0$

$\frac{\left(\beta R_{S I}+V_{S I}^{P A^{\prime}} \mu_{a}\right)\left\{2 \beta(1-\varphi) R_{S P}-\beta(1+\varphi) R_{S I}+\left[2(1-) V_{S P}^{P A^{\prime}}-(1+\varphi) V_{S I}^{P A^{\prime}}\right] \mu_{a}\right\}}{2(1-\varphi)^{3} k_{a}}=0$

Solving the linear Eqs. (12) and (13), we can obtain the values of $a_{1}, b_{1}, c_{1}$ and $d_{1}$ as follows:

$a_{1}=\frac{(1-\lambda) \theta+\gamma R_{S P}}{\rho+\mu_{g}} ; \quad c_{1}=\frac{\lambda \theta+\gamma R_{S I}}{\rho+\mu_{g}} ; \quad b_{1}=\frac{\left\{\begin{array}{c}4 k_{a}\left\{k_{s} \mu_{e}^{2}\left\{\theta(1-\lambda)+\gamma R_{S P}\right\}^{2}+\alpha^{2} k_{e} R_{S P}^{2}\left(\rho+\mu_{g}\right)^{2}\right\} \\ +k_{e} k_{S}\left\{\theta(2-\lambda) \mu_{a}+\left[\gamma \mu_{a}+\beta\left(\rho+\mu_{g}\right)\right]\left(2 R_{S P}+R_{S I}\right)\right\}^{2}\end{array}\right\}}{8 \rho k_{a} k_{e} k_{S}\left(\rho+\mu_{g}\right)^{2}}$

$d_{1}=\frac{\left\{\begin{array}{c}4 k_{a} \mu_{e}^{2}\left\{k_{S}\left[\theta(1-\lambda)+\gamma R_{m}\right]\left(\theta \lambda+\gamma R_{S I}\right)+\alpha^{2} k_{e} R_{S P} R_{S I}\left(\rho+\mu_{g}\right)^{2}\right\}+ \\ k_{e} k_{S}\left\{\theta \lambda \mu_{a}+R_{S I}\left[\gamma \mu_{a}+\beta\left(\rho+\mu_{g}\right)\right]\right\}\left\{\theta(2-\lambda) \mu_{a}+\left[\gamma \mu_{a}+\beta\left(\rho+\mu_{g}\right)\right]\left(2 R_{S P}+R_{S I}\right)\right\}\end{array}\right\}}{4 \rho k_{a} k_{e} k_{s}\left(\rho+\mu_{g}\right)^{2}}$ 
By substituting above equations into Eq. (11), we can get Lemma 1.

\section{Proof of Lemma 2}

Similar to Lemma 1, we omit the solving processes and just list the key values of parameters $a_{2}, b_{2}, c_{2}$ and $d_{2}$ as follows:

$a_{2}=\frac{\lambda \theta+\gamma R_{S I}}{\rho+\mu_{g}} ; \quad c_{2}=\frac{(1-\lambda) \theta+\gamma R_{S P}}{\rho+\mu_{g}}$

$b_{2}=\frac{\left\{\begin{array}{c}2 k_{a}\left\{k_{S}\left[\theta(1-\lambda)+\gamma R_{S P}\right]\left(\theta+\theta \lambda+\gamma R_{S P}+2 \gamma R_{S I}\right) \mu_{e}^{2}+2 \alpha^{2} k_{e} R_{S P}^{2}\left(\rho+\mu_{g}\right)^{2}\right\} \\ +k_{e} k_{S}\left\{\theta(2-\lambda) \mu_{a}+\left(2 R_{S P}+R_{S I}\right)\left[\gamma \mu_{a}+\beta\left(\rho+\mu_{g}\right)\right]\right\}^{2}\end{array}\right\}}{8 \rho k_{a} k_{e} k_{S}\left(\rho+\mu_{g}\right)^{2}}$

$d_{2}=\frac{\left\{\begin{array}{c}k_{a}\left[k_{S}\left(\theta+\theta \lambda+\gamma R_{S P}+2 \gamma R_{S I}\right)^{2} \mu_{e}^{2}+8 \alpha^{2} k_{e} R_{S P} R_{S I}\left(\rho+\mu_{g}\right)^{2}\right] \\ +2 k_{e} k_{S}\left\{\theta \lambda \mu_{a}+R_{S I}\left[\gamma \mu_{a}+\beta\left(\rho+\mu_{g}\right)\right]\right\}\left\{\theta(2-\lambda) \mu_{a}+\left[\gamma \mu_{a}+\beta\left(\rho+\mu_{g}\right)\right]\left(2 R_{S P}+R_{S I}\right)\right\}\end{array}\right\}}{8 \rho k_{a} k_{e} k_{S}\left(\rho+\mu_{g}\right)^{2}}$

The concrete expression of stationary emission reduction level is

$G_{\infty}^{P A I E *}=\frac{k_{a}\left(\theta+\theta \lambda+\gamma R_{S P}+2 \gamma R_{S I}\right) \mu_{e}^{2}+k_{e} \mu_{a}\left\{\theta(2-\lambda) \mu_{a}+\left(2 R_{S P}+R_{S I}\right)\left[\gamma \mu_{a}+\beta\left(\rho+\mu_{g}\right)\right]\right\}}{2 \gamma k_{a} k_{e}\left(\rho+\mu_{g}\right)}$

\section{Proof of Lemma 3}

Similarly, the parameters $a_{3}, b_{3}, c_{3}$ and $d_{3}$ are given as follows.

$a_{3}=\frac{(1-\lambda) \theta+\gamma R_{S P}}{\rho+\mu_{g}} ; \quad c_{3}=\frac{\lambda \theta+\gamma R_{S I}}{\rho+\mu_{g}}$

$b_{3}=\frac{\left\{\begin{array}{c}2 k_{a}\left\{2 k_{s} \mu_{e}^{2}\left[\theta(1-\lambda)+\gamma R_{S P}\right]^{2}+\alpha^{2} k_{e} R_{S P}\left(R_{S P}+2 R_{S I}\right)\left(\rho+\mu_{g}\right)^{2}\right\} \\ +k_{e} k_{S}\left\{\theta(2-\lambda) \mu_{a}+\left[\gamma \mu_{a}+\beta\left(\rho+\mu_{g}\right)\right]\left(2 R_{S P}+R_{S I}\right)\right\}^{2}\end{array}\right\}}{8 \rho k_{a} k_{e} k_{s}\left(\rho+\mu_{g}\right)^{2}}$

$d_{3}=\frac{\left\{\begin{array}{c}k_{a}\left\{8 k_{s} \mu_{e}^{2}\left[\theta(1-\lambda)+\gamma R_{S P}\right]\left(\theta \lambda+\gamma R_{S I}\right)+\alpha^{2} k_{e}\left(R_{S P}+2 R_{S I}\right)^{2}\left(\rho+\mu_{g}\right)^{2}\right\} \\ +2 k_{e} k_{S}\left\{\theta \lambda \mu_{a}+R_{S I}\left[\gamma \mu_{a}+\beta\left(\rho+\mu_{g}\right)\right]\right\}\left\{\theta(2-\lambda) \mu_{a}+\left[\gamma \mu_{a}+\beta\left(\rho+\mu_{g}\right)\right]\left(2 R_{S P}+R_{S I}\right)\right\}\end{array}\right\}}{8 \rho k_{a} k_{e} k_{S}\left(\rho+\mu_{g}\right)^{2}}$

The concrete expression of stationary emission reduction level is

$G_{\infty}^{P A I S *}=\frac{2 k_{a}\left(\theta-\theta \lambda+\gamma R_{S P}\right) \mu_{e}^{2}+k_{e} \mu_{a}\left\{\theta(2-\lambda) \mu_{a}+\left(2 R_{S P}++R_{S I}\right)\left[\gamma \mu_{a}+\beta\left(+\mu_{g}\right)\right]\right\}}{2 \gamma k_{a} k_{e}\left(\rho+\mu_{g}\right)}$

\section{Appendix B. Proofs of Propositions and Corollaries}

\section{Proof of Proposition 1}

It is easy to find that $S^{P A *}$ is independent of parameters $\theta, \lambda$ and $\gamma$, so we can get 
Proposition 1(i). In addition, taking first-order partial derivatives of $E^{P A *}, A^{P A *}$ and $\varphi^{P A *}$ with respect to $\theta, \lambda$ and $\gamma$ yields the following expressions.

$\frac{\partial E^{P A *}}{\partial \theta}=\frac{\mu_{e}(1-\lambda)}{k_{e}\left(\rho+\mu_{g}\right)}>0, \frac{\partial E^{P A *}}{\partial \lambda}=\frac{-\mu_{e} \theta}{k_{e}\left(\rho+\mu_{g}\right)}<0, \frac{\partial E^{P A *}}{\partial \gamma}=\frac{\mu_{e} R_{S P}}{k_{e}\left(\rho+\mu_{g}\right)}>0$

$\frac{\partial A^{P A *}}{\partial \theta}=\frac{(2-\lambda) \mu_{a}}{2 k_{a}\left(\rho+\mu_{g}\right)}>0, \frac{\partial A^{P A *}}{\partial \lambda}=\frac{-\theta \mu_{a}}{2 k_{a}\left(\rho+\mu_{g}\right)}<0, \frac{\partial A^{P A *}}{\partial \gamma}=\frac{2 R_{S P} \mu_{a}+R_{S I} \mu_{a}}{2 k_{a}\left(\rho+\mu_{g}\right)}>0$

$\frac{\partial \varphi^{P A *}}{\partial \theta}=\frac{-4\left[\lambda R_{S P}-(1-\lambda) R_{S I}\right] \mu_{a}\left[\gamma \mu_{a}+\beta\left(\rho+\mu_{g}\right)\right]}{\left\{\theta(2-\lambda) \mu_{a}+\left(2 R_{S P}+R_{S I}\right)\left[\gamma \mu_{a}+\beta\left(\rho+\mu_{g}\right)\right]\right\}^{2}}$

$\frac{\partial \varphi^{P A *}}{\partial \lambda}=\frac{-4 \theta \mu_{a}\left\{\theta \mu_{a}+\left[\gamma \mu_{a}+\beta\left(\rho+\mu_{g}\right)\right]\left(R_{S P}+R_{S I}\right)\right\}}{\left\{\theta(2-\lambda) \mu_{a}+\left[\gamma \mu_{a}+\beta\left(\rho+\mu_{g}\right)\right]\left(2 R_{S P}+R_{S I}\right)\right\}^{2}}$

$\frac{\partial \varphi^{P A *}}{\partial \gamma}=\frac{4 \theta\left(\lambda R_{S P}-(1-\lambda) R_{S I}\right) \mu_{a}^{2}}{\left\{\theta(2-\lambda) \mu_{a}+\left[\gamma \mu_{a}+\beta\left(\rho+\mu_{g}\right)\right]\left(2 R_{S P}+R_{S I}\right)\right\}^{2}}$

Hence, we can obtain Proposition 1 by discussing the sign of each expression in above equations.

\section{Proof of Proposition 2}

By observing the optimal carbon emission reduction, it is obvious that $\partial E^{P A I E *} / \partial \theta>0$, $\partial E^{P A I E *} / \partial \lambda>0$ and $\partial E^{P A I E *} / \partial \gamma>0$. Similar to Proposition 1, we have

$\frac{\partial \phi^{P A I E *}}{\partial \theta}=\frac{4 \gamma\left[\lambda R_{S P}-(1-\lambda) R_{S I}\right]}{\left(\theta+\theta \lambda+\gamma R_{S P}+2 \gamma R_{S I}\right)^{2}}, \frac{\partial \phi^{P A I E *}}{\partial \lambda}=\frac{4 \theta\left(\theta+\gamma R_{S P}+\gamma R_{S I}\right)}{\left(\theta+\theta \lambda+\gamma R_{S P}+2 \gamma R_{S I}\right)^{2}}>0$

$\frac{\partial \phi^{P A I E *}}{\partial \gamma}=\frac{-4 \theta\left[\lambda R_{S P}-(1-\lambda) R_{S I}\right]}{\left(\theta+\theta \lambda+\gamma R_{S P}+2 \gamma R_{S I}\right)^{2}}$

Hence, we can obtain Proposition 2 by discussing the sign of each expression in above equations.

\section{Proof of Proposition 3}

Lemma 2 gives $\quad \varphi^{*}=\frac{\theta(2-3 \lambda) \mu_{a}+\left(2 R_{S P}-R_{S I}\right)\left[\gamma \mu_{a}+\beta\left(\rho+\mu_{g}\right)\right]}{\theta(2-\lambda) \mu_{a}+\left(2 R_{S I}+R_{S P}\right)\left[\gamma \mu_{a}+\beta\left(\rho+\mu_{g}\right)\right]} ; \quad \phi^{*}=\frac{\gamma\left(2 R_{S I}-R_{S P}\right)-\theta(1-3 \lambda)}{\theta(1+\lambda)+\gamma\left(2 R_{S I}+R_{S P}\right)}$. By discussing the signs of $\varphi^{*}$ and $\phi^{*}$, we can easily obtain the Proposition 3.

\section{Proof of Corollary 1}

According to Lemma 2, making difference between $\varphi^{P A I E *}$ and $\phi^{P A I E *}$ yields the following equation.

$$
\varphi^{P A I E *}-\phi^{P A I E *}=\frac{\left\{\begin{array}{c}
4\left\{\theta^{2}(1-2 \lambda) \mu_{a}+\left(R_{S P}^{2}-R_{S I}^{2}\right) \gamma\left[\gamma \mu_{a}+\beta\left(\rho+\mu_{g}\right)\right]\right. \\
\left.+\theta(1-\lambda) R_{S P}\left[2 \gamma \mu_{a}+\beta\left(\rho+\mu_{g}\right)\right]+\theta \lambda R_{S I}\left[2 \gamma \mu_{a}+\beta\left(\rho+\mu_{g}\right)\right]\right\}
\end{array}\right\}}{\left(\theta+\theta \lambda+\gamma R_{S P}+2 \gamma R_{S I}\right)\left\{\theta(2-\lambda) \mu_{a}+\left[\gamma \mu_{a}+\beta\left(\rho+\mu_{g}\right)\right]\left(2 R_{S P}+R_{S I}\right)\right\}}
$$


Therefore, when $0<\lambda \leq \min \left(\bar{\lambda}_{1}, 1\right)$, we have $\varphi^{P A I E *} \geq \phi^{P A I E *}$, and vice versa.

Proof of Proposition 4 is similar to Proposition 3.

Proof of Corollary 2 is similar to Corollary 1.

\section{Proof of Proposition 5}

It can be easily found that $S^{P A *}=S^{P A I E *}, E^{P A *}=E^{P A I S *}$ and $A^{P A *}=A^{P A I E *}=A^{P A I S *}$. Comparisons of the services and emission reductions under contracts PAIE and PAIS lead to the following expressions.

$$
S^{P A I E *}-S^{P A I S *}=\frac{\alpha\left(R_{S P}-2 R_{S I}\right)}{2 k_{S}}, E^{P A I E *}-E^{P A I S *}=\frac{\left[\theta(1-3 \lambda)+\gamma\left(R_{S P}-2 R_{S I}\right)\right] \mu_{e}}{-2 k_{e}\left(\rho+\mu_{g}\right)}
$$

Consequently, discussing the signs of the above expressions yields Proposition 5.

\section{Proof of Corollary 3}

By taking the difference among emission reduction levels across the three cost-sharing contracts, we can have the following expressions: $G^{P A *}-G^{\text {PAIS* }}=0, G^{\text {PAIS* }}-G^{\text {PAIE* }}=$ $\frac{\left[\theta(1-3 \lambda)+\gamma\left(R_{S P}-2 R_{S I}\right)\right] \mu_{e}^{2}}{2 \gamma k_{e}\left(\rho+\mu_{g}\right)}$. Hence, it is easy to derive the corollary by discussing the signs of these expressions.

\section{Proof of Proposition 6}

Similar to Proposition 5, we have $\phi^{\text {PAIE* }}-\phi^{P A I S *}=\frac{4 \theta\left[\lambda R_{S P}-(1-\lambda) R_{S I}\right]}{\left(R_{S P}+2 R_{S I}\right)\left(\theta+\theta \lambda+\gamma R_{S P}+2 \gamma R_{S I}\right)}$. When $\lambda R_{S P} \geq(1-\lambda) R_{S I}$, we can obtain $\phi^{P A I E *} \geq \phi^{P A I S *}$, and vice versa.

\section{Proof of Proposition 7}

Comparing the SP's optimal utilities in the three cost-sharing contracts, we can have the following expressions.

$$
\begin{aligned}
& U_{S P}^{P A I E *}-U_{S P}^{P A *}=\frac{\left[\theta(1-\lambda)+\gamma R_{S P}\right]\left[\gamma\left(2 R_{S I}-R_{S P}\right)-\theta(1-3 \lambda)\right] \mu_{e}^{2}}{4 k_{e}\left(\rho+\mu_{g}\right)^{2}} \\
& U_{S P}^{P A I S *}-U_{S P}^{P A I E *}=\frac{\left\{\alpha^{2} k_{e} R_{S P}\left(2 R_{S I}-R_{S P}\right)\left(\rho+\mu_{g}\right)^{2}-k_{s}\left[\theta(1-\lambda)+\gamma R_{S P}\right]\left[\gamma\left(2 R_{S I}-R_{S P}\right)-\theta(1-3 \lambda)\right] \mu_{e}^{2}\right\}}{4 k_{e} k_{S}\left(\rho+\mu_{g}\right)^{2}} \\
& U_{S P}^{P A I S *}-U_{S P}^{P A *}=\frac{\alpha^{2} R_{S P}\left(2 R_{S I}-R_{S P}\right)}{4 k_{S}}
\end{aligned}
$$


By discussing the signs of the above expressions, the proposition can be completely verified.

\section{Proof of Proposition 8}

Similar to Proposition 7, we here provide the corresponding difference value expressions as follows.

$$
\begin{aligned}
& U_{S I}^{P A I E *}-U_{S I}^{P A *}=\frac{\left[\theta(1-3 \lambda)+\gamma\left(R_{S P}-2 R_{S I}\right)\right]^{2} \mu_{e}^{2}}{8 k_{e}\left(\rho+\mu_{g}\right)^{2}}, U_{S I}^{P A I S *}-U_{S I}^{P A *}=\frac{\alpha^{2}\left(R_{S P}-2 R_{S I}\right)^{2}}{8 k_{S}} \\
& U_{S I}^{P A I S *}-U_{S I}^{P A I E *}=\frac{\alpha^{2} k_{e}\left(R_{S P}-2 R_{S I}\right)^{2}\left(\rho+\mu_{g}\right)^{2}-k_{S}\left(\theta-3 \theta \lambda+\gamma R_{S P}-2 \gamma R_{S I}\right)^{2} \mu_{e}^{2}}{8 k_{e} k_{S}\left(\rho+\mu_{g}\right)^{2}}
\end{aligned}
$$

\section{Proof of Corollary 4}

Making a difference between the thresholds $\bar{k}_{s 1}$ and $\bar{k}_{s 2}$ yields the following result.

$$
\bar{k}_{S 1}-\bar{k}_{S 2}=\frac{2 \alpha^{2} \theta k_{e}\left(2 R_{S I}-R_{S P}\right)\left[\lambda R_{S P}-(1-\lambda) R_{S I}\right]\left(\rho+\mu_{g}\right)^{2}}{\left[\theta(1-\lambda)+\gamma R_{S P}\right]\left[\theta(1-3 \lambda)-2 \gamma R_{S I}+\gamma R_{S P}\right]^{2} \mu_{e}^{2}}
$$

Based on above equation and Propositions 7 and 8, we can derive the Corollary 4 by finding out the same parameter regions under which SP and SI have the same preference for the optimal contract.

\section{Proof of Proposition 9}

Similarly, we here provide the corresponding comparative results as follows:

$$
\begin{aligned}
& U_{S C}^{P A I E *}-U_{S C}^{P A *}=\frac{\left[\gamma\left(2 R_{S I}-R_{S P}\right)-\theta(1-3 \lambda)\right]\left[\theta(1+\lambda)+\gamma\left(R_{S P}+2 R_{S I}\right)\right] \mu_{e}^{2}}{8 k_{e}\left(\rho+\mu_{g}\right)^{2}} \\
& U_{S C}^{P A I S *}-U_{S C}^{P A I E *}=\frac{\left\{\begin{array}{c}
k_{S}\left\{\theta^{2}\left(1-2 \lambda-3 \lambda^{2}\right)+2 \gamma \theta\left[(1-\lambda) R_{S P}-4 \lambda R_{S I}\right]+\gamma^{2}\left(R_{S P}^{2}-4 R_{S I}^{2}\right)\right\} \mu_{e}^{2} \\
-\alpha^{2} k_{e}\left(R_{S P}^{2}-4 R_{S I}^{2}\right)\left(\rho+\mu_{g}\right)^{2}
\end{array}\right\}}{8 k_{e} k_{S}\left(\rho+\mu_{g}\right)^{2}} \\
& U_{S C}^{P A I S *}-U_{S C}^{P A *}=\frac{\alpha^{2}\left(4 R_{S I}^{2}-R_{S P}^{2}\right)}{8 k_{S}}
\end{aligned}
$$

Proof of Proposition 10 is similar to Propositions 7-9.

\section{References}

Achtnicht, M. (2012). German car buyers' willingness to pay to reduce co emissions. Climatic Change, 113(3-4), 679-697. 
Agan, Y., Kuzey, C., Acar, M. F., \& Acikgoz, A. (2016). The relationships between corporate social responsibility, environmental supplier development, and firm performance. Journal of Cleaner Production, 112(3), 1872-1881.

Andersen, M., \& Skjoett-Larsen, T. (2009). Corporate social responsibility in global supply chains. Supply Chain Management: An International Journal, 14(2), 75-86.

Arya, A., \& Mittendorf, B. (2015). Supply chain consequences of subsidies for corporate social responsibility. Production \& Operations Management, 24(8), 1346-1357.

Bai, Q., Chen, M., \& Xu, L. (2017). Revenue and promotional cost-sharing contract versus two-part tariff contract in coordinating sustainable supply chain systems with deteriorating items. International Journal of Production Economics, 187, 85-101.

Balachandran, K. R., \& Radhakrishnan, S. (2005). Quality implications of warranties in a supply chain. Management Science, 51(8), 1266-1277.

Banerjee, S., \& Wathieu, L. (2017). Corporate social responsibility and product quality: complements or substitutes?. International Journal of Research in Marketing, 34(3), 734-745.

Bazan, E., Jaber, M. Y., \& Zanoni, S. (2016). Carbon emissions and energy effects on a two-level manufacturer-retailer closed-loop supply chain model with remanufacturing subject to different coordination mechanisms. International Journal of Production Economics, 183, 394-408.

Cachon, G. P., \& Lariviere, M. A. (2005). Supply chain coordination with revenue-sharing contracts: strengths and limitations. Management Science, 51(1), 30-44.

Cao, E., \& Yu, M. (2018). Trade credit financing and coordination for an emission-dependent supply chain. Computers \& Industrial Engineering, 119, 50-62.

Cellini, R., \& Lambertini, L. (2004). Dynamic oligopoly with sticky prices: closed-loop, feedback, and open-loop solutions. Journal of Dynamical \& Control Systems, 10(3), 303-314.

Chutani, A., \& Sethi, S. P. (2018). Dynamic cooperative advertising under manufacturer and retailer level competition. European Journal of Operational Research, 268, 635-652. 
Cruz, J. M. \& Matsypura, D. (2009). Supply chain networks with corporate social responsibility through integrated environmental decision-making. International Journal of Production Research, 47(3), 621-648.

Eisingerich, A. B., Rubera, G., Seifert, M., \& Bhardwaj, G. (2011). Doing good and doing better despite negative information?: the role of corporate social responsibility in consumer resistance to negative information. Journal of Service Research, 14(1), 60-75.

Frisk, M., Göthe-Lundgren, M., Jörnsten, K., \& Rönnqvistab, M. (2010). Cost allocation in collaborative forest transportation. European Journal of Operational Research, 205(2), $448-458$.

Ghosh, D., \& Shah, J. (2015). Supply chain analysis under green sensitive consumer demand and cost sharing contract. International Journal of Production Economics, 164, 319-329.

Han, H., \& Yoon, H. J. (2015). Hotel customers' environmentally responsible behavioral intention: impact of key constructs on decision in green consumerism. International Journal of Hospitality Management, 45, 22-33.

He, P., He, Y., \& Xu, F. (2018). Evolutionary analysis of sustainable tourism. Annals of Tourism Research, 69, 76-89.

He, P., He, Y., Xu, F., \& Shi, C. (2019). Sustainable tourism modeling: Pricing decisions and evolutionarily stable strategies for competitive tour operators. Tourism Economics, 25(5), 779-799.

He, X., Prasad, A., \& Sethi, S. P. (2009). Cooperative advertising and pricing in a dynamic stochastic supply chain: feedback Stackelberg strategies. Production \& Operations Management, 18(1), 78-94.

Hsueh, C. F. (2014). Improving corporate social responsibility in a supply chain through a new revenue sharing contract. International Journal of Production Economics, 151(3), 214-222.

Hsueh, C. F., \& Chang, M. S. (2008). Equilibrium analysis and corporate social responsibility for supply chain integration. European Journal of Operational Research, 190(1), 116-129. 
Ji, J., Zhang, Z., \& Yang, L. (2017). Carbon emission reduction decisions in the retail-/dual-channel supply chain with consumers' preference. Journal of Cleaner Production, 141, 852-867.

Jorgensen, S., Taboubi, S., \& Zaccour, G. (2001). Cooperative advertising in a marketing channel. Journal of Optimization Theory \& Applications, 110(1), 145-158.

Lee, H. L., \& Tang, C. S. (2017). Socially and environmentally responsible value chain innovations: new operations management research opportunities. Management Science, 64(3), 983-996.

Letizia, P., \& Hendrikse, G. (2016). Supply chain structure incentives for corporate social responsibility: an incomplete contracting analysis. Production \& Operations Management, 25(11), 1919-1941.

Liu, K. \& Song, H. (2017). Contract and incentive mechanism in low-carbon R\&D cooperation. Supply Chain Management: An International Journal, 22(3), 270-283.

Liu, W. H., Xu, X. C., \& Kouhpaenejad, A. (2013). Deterministic approach to the fairest revenue-sharing coefficient in logistics service supply chain under the stochastic demand condition. Computers \& Industrial Engineering, 66(1), 41-52.

Liu, W., Wang, S., Zhu, D. L., Wang, D., \& Shen, X. (2017). Order allocation of logistics service supply chain with fairness concern and demand updating: model analysis and empirical examination. Annals of Operations Research, 268(2), 1-37.

Liu, Y., Quan, B. T., Li, J., \& Forrest, J. (2018). A supply chain coordination mechanism with cost sharing of corporate social responsibility. Sustainability, 10(4), 1227.

Liu, Z., Anderson, T. D., \& Cruz, J. M. (2012). Consumer environmental awareness and competition in two-stage supply chains. European Journal of Operational Research, 218(3), 602-613.

Lombart, C., \& Louis, D. (2014). A study of the impact of corporate social responsibility and price image on retailer personality and consumers' reactions (satisfaction, trust and loyalty to the retailer). Journal of Retailing \& Consumer Services, 21(4), 630-642.

Luo, Z., Chen, X., \& Wang, X. (2016). The role of co-opetition in low carbon manufacturing. 
European Journal of Operational Research, 253(2), 392-403.

Panda, S., \& Modak, N. M. (2016). Exploring the effects of social responsibility on coordination and profit division in a supply chain. Journal of Cleaner Production, 139, 25-40.

Panda, S., Modak, N. M., \& Cardenas-Barron, L. E. (2017). Coordinating a socially responsible closed-loop supply chain with product recycling. International Journal of Production Economics, 188, 11-21.

Piga, C. A. G. (2000). Competition in a duopoly with sticky price and advertising. International Journal of Industrial Organization, 18(4), 595-614.

Sayed, M., Hendry, L. C. \& Bell, M. Z. (2017). Institutional complexity and sustainable supply chain management practices. Supply Chain Management: An International Journal, 22(6), $542-563$.

Shen, B., Ding, X., Chen, L., Chan, H. L., Govindan, K., \& Wagner, B. (2017). Low carbon supply chain with energy consumption constraints: case studies from china's textile industry and simple analytical model. Supply Chain Management: An International Journal, 22(3), $258-269$.

Tsay, A. (1999). The quantity flexibility contract and supplier-customer incentives. Management Science, 45(10), 1339-1358.

Wang, K., Wei, Y. M., \& Huang, Z. (2016). Potential gains from carbon emissions trading in China: A DEA based estimation on abatement cost savings. Omega, 63, 48-59.

Wu, Y., Li, H., Gou, Q., \& Gu, J. (2017). Supply chain models with corporate social responsibility. International Journal of Production Research, 55(3), 1-28.

Xia, L., Guo, T., Qin, J., Yue, X., \& Zhu, N. (2018). Carbon emission reduction and pricing policies of a supply chain considering reciprocal preferences in cap-and-trade system. Annals of Operations Research, 268, 149-175.

Xu, X., He, P., Xu, H., \& Zhang, Q. (2017). Supply chain coordination with green technology under cap-and-trade regulation. International Journal of Production Economics, 183, 433-442. 
Yang, F., Shan, F., \& Jin, M. (2017). Capacity investment under cost sharing contracts. International Journal of Production Economics, 191, 278-285.

Yang, H., \& Chen, W. (2018). Retailer-driven carbon emission abatement with consumer environmental awareness and carbon tax: revenue-sharing versus cost-sharing. Omega, 78, 179-191.

Yang, L., Ji, J., Wang, M., \& Wang, Z. (2018). The manufacturer's joint decisions of channel selections and carbon emission reductions under the cap-and-trade regulation. Journal of Cleaner Production, 193, 506-523.

Yoon, Y., Gürhan-Canli, Z., \& Schwarz, N. (2006). The effect of corporate social responsibility (CSR) activities on companies with bad reputations. Journal of Consumer Psychology, 16(4), 377-390.

Yuen, K. F., Thai, V. V., Wong, Y. D., \& Wang, X. (2018). Interaction impacts of corporate social responsibility and service quality on shipping firms' performance. Transportation Research Part A Policy \& Practice, 113, 397-409.

Zhang, J., Gou, Q., Liang, L., \& Huang, Z. (2013). Supply chain coordination through cooperative advertising with reference price effect. Omega, 41(2), 345-353.

Zhang, J., Lei, L., Zhang, S., \& Song, L. (2017). Dynamic vs. static pricing in a supply chain with advertising. Computers \& Industrial Engineering, 109, 266-279.

Zhang, S., Wang, C., Yu, C., \& Ren, Y. (2019). Governmental cap regulation and manufacturer's low carbon strategy in a supply chain with different power structures. Computers \& Industrial Engineering, 134, 27-36.

Zhang, Y., Hafezi, M., Zhao, X., \& Shi, V. (2017). The impact of development cost on product line design and its environmental performance. International Journal of Production Economics, $184,122-130$.

Zhao, L., Zha, Y., Wei, K., \& Liang, L. (2017). A target-based method for energy saving and carbon emissions reduction in china based on environmental data envelopment analysis. Annals of 
Operations Research, 255(1-2), 1-24.

Zhou, Y. W., Guo, J., \& Zhou, W. (2018). Pricing/service strategies for a dual-channel supply chain with free riding and service-cost sharing. International Journal of Production Economics, 196, 198-210.

Zhou, Y., \& Ye, X. (2018). Differential game model of joint emission reduction strategies and contract design in a dual-channel supply chain. Journal of Cleaner Production, 190, 592-607.

Zhou, Y., Bao, M., Chen, X., \& Xu, X. (2016). Co-op advertising and emission reduction cost sharing contracts and coordination in low-carbon supply chain based on fairness concerns. Journal of Cleaner Production. 133, 402-413.

Zu, Y., Chen, L., \& Fan, Y. (2018). Research on low-carbon strategies in supply chain with environmental regulations based on differential game. Journal of Cleaner Production, 177, $527-546$. 


\title{
Detailed Response to Reviewers
}

\author{
Ref. No.: CAIE-D-19-00620
}

Title: Cost-sharing contract design in a low-carbon service supply chain Computers \& Industrial Engineering

Dear Editors and Reviewers:

Thank you very much for your letter and for the reviewers' comments concerning our manuscript entitled "Cost-sharing contract design in a low-carbon service supply chain". We believe the revised paper after considering your advice has been significantly improved and we hope it is now publishable.

We have provided our detailed point-to-point responses below. We look forward to hearing from you.

Yours sincerely,

The authors 


\section{Responses to Reviewer 1's Comments}

This paper studied a cost allocation problem in a service supply chain (SC) that was consisting of a service provider (SP) and a service integrator (SI). The paper was written clearly. However, the following comments needs to be replied before considering of publication.

Response: Thank you for the good comments! We have carefully studied them and made some changes and explanations point-by-point as below.

\section{Comments :}

1. The situation considered in this paper is relatively simple as the service supply chain has just a service provider and a service integrator. Whether the results of this paper can be extended to a more complex supply chain with multiple service providers and integrators?

Response: Thanks for your question which considers a more complex business situation. There are some reasons why we choose a simple supply chain involving one service provider and one service integrator. First, the focus of our study is on the vertical partnership between the service provider and the service integrator instead of the horizontal competition among peers. Second, by focusing on the simple supply chain as mentioned in main text, we can obtain analytical solutions and more specific managerial insights. Third, this simple supply chain structure has been extensively used in the literature of supply chain coordination (e.g., Jin et al., 2015; Liu et al., 2016; Panda et al., 2017; Xia et al., 2018; Zhou et al., 2016; Zu et al., 2018).

Additionally, as we all known that the horizontal competition often impacts the market shares of the individual firms that compete each other at the same level. Most studies on complex or competitive supply chains have assumed that the competitors are symmetric for clarity (the same system parameters), thereby leading to the same outcomes such as equal decision variables, demands and profits for all horizontal competitive firms ( $\mathrm{Li}$ and Li, 2016; Wu and Huang, 2018; Xia et al., 2019). Similarly, when considering multiple service providers and/or integrators in our study, we could derive that the total market share would be divided into several equivalent segments 
after complete competition (e.g., the Classical Bertrand Model). That means a single supply chain would get a smaller market share. Hence, it could be derived that the horizontal competition may change our research findings from the perspective of market share. However, our main research results related to contract design would still hold to a large extent because these findings are obtained by comparing the three cost-sharing contracts under the same market environment. In other words, the horizontal competition may impact our study results quantitatively but not essentially as it mainly impacts the external relationship of a supply chain (i.e., market equilibrium environment) instead of its internal relationship.

Based on the above discussions and following many prior studies, our paper focuses closely on a simple supply chain to address the issue of vertical contract design.

2. There was a strong assumption that the completely symmetric information was shared among collaborators. This is hardly applied in practice because some private information must be reserved by cooperative members. Thus, a cooperation mechanism is required to coordinate the information sharing and the sustainability of the cooperation. And the incentive of providing information for collaborators also needs to be discussed.

Response: As explained above, in this study, we focus on vertical contract design rather than other issues such as information sharing. Hence, we assume that information is symmetric for every participant. This assumption has been broadly adopted in many prior studies (e.g., Ghosh and Shah, 2012; Huang et al., 2013; Savaskan and Van Wassenhove, 2006; Xia et al., 2018; Zhang et al., 2017). The classical Bertrand and Cournot models are also built on this assumption, which is reasonable in some real situations. This is the possible case where the upstream and downstream or competitive firms are willing to share their operational information such as inventory information with each other to enable better cooperation. For example, Genghis Khan Airlines and Chengdu Airlines have signed a cooperation framework agreement that includes sharing of operational information to facilitate the ARJ21 aircraft model entering commercial operation at scale (http://www.globaltimes.cn/content/1160009.shtml). In this setting, it is unnecessary 
to worry about the information sharing issue as all of the players can benefit from their cooperation.

Even if there is a risk of players sharing the wrong information, the supply chain members can also sign a penalty mechanism contract to avoid information rent-seeking issue. However, the information rent-seeking issue is beyond the scope of our current study. Of course, we also think the incentive of information sharing or private information is an interesting and professional issue so that we have listed it as a future study direction (Please see the fifth paragraph in Section 7).

Thanks again for your help in the reviewing process. We hope our new revision is satisfactory and publishable.

\section{Responses to Reviewer 2's Comments}

This work studies the cost-sharing contract design in a low-carbon service supply chain. Three different game models are studied to explore the optimal decisions, and identify the conditions under which provider and integrator would provide positive participation rates. Overall, this work is well written. While the setting is not completely new, this work could be a positive addition to existing literature. I recommend it for publication.

Response: Thank you very much for your positive comments and suggestions. We hope our new revision is satisfactory and publishable.

\section{References}

Ghosh, D., \& Shah, J. (2012). A comparative analysis of greening policies across supply chain structures. International Journal of Production Economics, 135(2), 568-583. 
Huang, M., Song, M., Lee, L.H., \& Ching, W.K. (2013). Analysis for strategy of closed-loop supply chain with dual recycling channel. International Journal of Production Economics, 144(2), 510-520.

Jin, Y., Wang, S., \& Hu, Q. (2015). Contract type and decision right of sales promotion in supply chain management with a capital constrained retailer. European Journal of Operational Research, 240(2), 415-424.

Li, X., \& Li, Y. (2016). Chain-to-chain competition on product sustainability. Journal of Cleaner Production, 112, 2058-2065.

Liu, G., Sethi, S.P., \& Zhang, J. (2016). Myopic vs. far-sighted behaviours in a revenue-sharing supply chain with reference quality effects. International Journal of Production Research, 54(5), 1-24.

Panda, S., Modak, N.M., \& Cárdenas-Barrón, L.E. (2017). Coordinating a socially responsible closed-loop supply chain with product recycling. International Journal of Production Economics, 188, 11-21.

Savaskan, C., \& Van Wassenhove, L.N., 2006. Reverse channel design: the case of competing retailers. Management Science 52 (1), 1-14.

Wu, J., \& Huang, Q. (2018). Myopic and far-sighted pricing strategies in a duopoly market with e-WOM effect. International Journal of Electronic Commerce, 22:4, 609-630.

Xia, L., Guo, T., Qin, J., Yue, X., \& Zhu, N. (2018). Carbon emission reduction and pricing policies of a supply chain considering reciprocal preferences in cap-and-trade system. Annals of Operations Research, 268, 149-175.

Xia, Y., Xiao, T., \& Zhang, G. P. (2019). Service investment and channel structure decisions in competing supply chains. Service Science, 11(1), 57-74.

Zhang, J., Lei, L., Zhang, S., \& Song, L. (2017). Dynamic vs. static pricing in a 
supply chain with advertising. Computers \& Industrial Engineering, 109, 266279.

Zhao, X., \& Shi, C. (2011). Structuring and contracting in competing supply chains. International Journal of Production Economics, 134(2), 434-446.

Zhou, Y., Bao, M., Chen, X., \& Xu, X. (2016). Co-op advertising and emission reduction cost sharing contracts and coordination in low-carbon supply chain based on fairness concerns. Journal of Cleaner Production, 133, 402-413.

Zu, Y., Chen, L., \& Fan, Y. (2018). Research on low-carbon strategies in supply chain with environmental regulations based on differential game. Journal of Cleaner Production, 177, 527-546. 